\title{
A!
}

This is an electronic reprint of the original article.

This reprint may differ from the original in pagination and typographic detail.

Filippov, S. N.; Piilo, J.; Maniscalco, S.; Ziman, M.

\section{Divisibility of quantum dynamical maps and collision models}

Published in:

Physical Review A

DOI:

10.1103/PhysRevA.96.032111

Published: 14/09/2017

Document Version

Publisher's PDF, also known as Version of record

Please cite the original version:

Filippov, S. N., Piilo, J., Maniscalco, S., \& Ziman, M. (2017). Divisibility of quantum dynamical maps and collision models. Physical Review A, 96(3), 1-13. [032111]. https://doi.org/10.1103/PhysRevA.96.032111

This material is protected by copyright and other intellectual property rights, and duplication or sale of all or part of any of the repository collections is not permitted, except that material may be duplicated by you for your research use or educational purposes in electronic or print form. You must obtain permission for any other use. Electronic or print copies may not be offered, whether for sale or otherwise to anyone who is not an authorised user. 


\title{
Divisibility of quantum dynamical maps and collision models
}

\author{
S. N. Filippov, ${ }^{1,2}$ J. Piilo, ${ }^{3}$ S. Maniscalco,${ }^{3,4}$ and M. Ziman ${ }^{5,6}$ \\ ${ }^{1}$ Moscow Institute of Physics and Technology, Institutskii Per. 9, Dolgoprudny, Moscow Region 141700, Russia \\ ${ }^{2}$ Institute of Physics and Technology, Russian Academy of Sciences, Nakhimovskii Prospekt 34, Moscow 117218, Russia \\ ${ }^{3}$ Turku Centre for Quantum Physics, Department of Physics and Astronomy, University of Turku, FI-20014 Turun Yliopisto, Finland \\ ${ }^{4}$ Centre for Quantum Engineering, Department of Applied Physics, School of Science, Aalto University, \\ P.O. Box 11000, FI-00076 Aalto, Finland \\ ${ }^{5}$ Institute of Physics, Slovak Academy of Sciences, Dúbravská cesta 9, Bratislava 84511, Slovakia \\ ${ }^{6}$ Faculty of Informatics, Masaryk University, Botanická 68a, Brno 60200, Czech Republic
}

(Received 17 August 2017; published 14 September 2017)

\begin{abstract}
The divisibility of dynamical maps is visualized by trajectories in the parameter space and analyzed within the framework of collision models. We introduce ultimate completely positive (CP) divisible processes, which lose $\mathrm{CP}$ divisibility under infinitesimal perturbations, and characterize Pauli dynamical semigroups exhibiting such a property. We construct collision models with factorized environment particles, which realize additivity and multiplicativity of generators of CP divisible maps. A mixture of dynamical maps is obtained with the help of correlated environment. The mixture of ultimate $\mathrm{CP}$ divisible processes is shown to result in a class of eternal $\mathrm{CP}$ indivisible evolutions. We explicitly find collision models leading to weakly and essentially non-Markovian Pauli dynamical maps.
\end{abstract}

DOI: 10.1103/PhysRevA.96.032111

\section{INTRODUCTION}

The theory of open quantum systems studies dynamical maps $\Phi_{t}$ that naturally occur when the system in question interacts with its environment. Dynamical maps are the key objects in the analysis of quantum information transmission through noisy channels [1] and quantum information processing in real systems [2]. The effect of open system dynamics on quantum entanglement and entanglement-based information protocols is reviewed, e.g., in Ref. [3]. Over the last decade, quantum dynamical maps were intensively studied with respect to characterization of their non-Markovian behavior [4-6] and its experimental observation [7-13]. Quantitative approaches to non-Markovianity include nonmonotonic distinguishability of states [14,15], different divisibilities of dynamical maps [16,17], monitoring the volume of accessible states [18], and others [19-22]. In particular, the divisibility approach is based on the decomposition property $\Phi_{t+s}=\Theta_{t, t+s} \Phi_{t}$ and explores features of the intermediate map $\Theta_{t, t+s}$ [23]. Various types of divisibility induce alternative measures to quantify non-Markovianity; however, one should be careful with the physical interpretation of memory effects [24].

From a mathematical viewpoint, the open system dynamics in the Schrödinger picture is given by the transformation $\varrho(t)=\Phi_{t}[\varrho(0)]$, where $\Phi_{t}$ is the dynamical map (process) that is a one-parameter family of completely positive tracepreserving (CPT) maps, $t \geqslant 0$ is the evolution time, and $\Phi_{0}=$ Id is the identity transformation. The dilation of the dynamical map is

$$
\Phi_{t}[\varrho]=\operatorname{tr}_{\mathrm{env}}\left\{U_{t}(\varrho \otimes \xi) U_{t}^{\dagger}\right\},
$$

where $U_{t}$ is the unitary evolution of the system and the environment, and $\xi$ is the initial state of the environment.

Complete positivity (CP) of $\Phi_{t}$ means that the map $\Phi_{t} \otimes$ $\operatorname{Id}_{k}$ is positive for all identity transformations $\operatorname{Id}_{k}$ of $k$-level ancillary systems, which can be potentially entangled with the system in question. If $\Theta_{t, t+s}$ is CP for all $t$ and $s \geqslant 0$, then the process $\Phi_{t}$ is called $C P$ divisible. Such a definition of CP divisibility is a global-in-time property of the whole family $\left\{\Phi_{t}\right\}_{t \geqslant 0}$. In contrast, to underline the time-local behavior, we will refer to a process $\Phi_{t}$ as CP divisible at time $t_{0}$ if there exists $s_{0}>0$ such that $\Theta_{t_{0}, t_{0}+s}$ is CP for all $s \in\left(0, s_{0}\right)$. If the dynamical map $\Phi_{t}$ is not CP divisible for all time moments $t \geqslant$ 0 , then $\Phi_{t}$ is called eternal CP indivisible [25]. CP divisibility of a bijective dynamical map was shown to be equivalent to the distinguishability of states in the extended Hilbert space [26].

Replacing CP by any other property [viz. positivity (P), $k$ positivity, volume of accessible states, etc.] we obtain definitions of the global and time-local divisibility properties of the dynamical map $\Phi_{t}$. Processes which are not CP divisible but are $\mathrm{P}$ divisible are also called weakly non-Markovian, whereas $\mathrm{P}$ indivisible processes are called essentially nonMarkovian [27].

Since any linear map $\Phi$ between finite-dimensional spaces can be defined by a set of real parameters $\lambda=\lambda_{1}, \ldots, \lambda_{n}$, any smooth process $\Phi_{t}$ is then determined by a continuous trajectory $\lambda(t)$ in the parameter space. Such a trajectory provides a pictorial representation of the dynamical map in $\mathbb{R}^{n}$, which is particularly visual in the case of qubit Pauli maps given by three parameters (see, e.g., [25,28-31]). Analyzing the process trajectory in the parameter space, one gets not only an intuition about the quantum dynamics (for instance, by observing the Bloch ball transformation for qubit dynamics), but it also reveals its divisibility properties. The first goal of this paper is to describe different forms of Markovian and non-Markovian Pauli dynamical maps in terms of trajectories in the parameter space.

A pictorial representation of some dynamical map $\Phi_{t}$ in the form of trajectory $\lambda(t)$ raises a question of the stability of the process with respect to a continuous (infinitely differentiable) trajectory perturbation $\lambda(t) \rightarrow \lambda(t)+\delta \lambda(t)$, with the perturbed map $\Phi_{t}+\delta \Phi_{t}$ being a valid quantum dynamical evolution. A process $\Phi_{t}$, which is originally CP divisible at time $t_{0}$, may lose the property of being $\mathrm{CP}$ divisible at 
this time due to a time-local perturbation $\delta \lambda(t)$ such that $\delta \lambda(t)=0$ if $t \leqslant t_{0}$. If this is the case, $\Phi_{t}$ is called ultimate CP divisible at time $t_{0}$. There exist processes $\Phi_{t}$ that are ultimate CP divisible for all time moments $t \geqslant 0$. We fully characterize Pauli dynamical semigroups exhibiting such a property.

In this paper, we show that the mathematical concepts of divisibility are closely related with the underlying physical models of quantum dynamical maps. From a physical viewpoint, any dynamical map $\Phi_{t}$ can be seen as a simplified description of the system-environment evolution with no regard to the environment structure and particular microscopic interactions between environment quanta and the system. Many dynamical maps can be derived under some assumptions (weak coupling, low density, etc.) from a microscopic system-environment Hamiltonian and particular state of the environment $[6,32]$. In our analysis, we will resort to the so-called collision models in which the motional and internal degrees of freedom can be considered separately: the motion Hamiltonian determines a sequence of collisions with environment particles, and the system-environment interaction Hamiltonian becomes significant during collisions and affects internal degrees of freedom of the system and an impacted environment particle. A relaxation mechanism via such a "stirring" process was first considered in Ref. [33]. Thermalization, homogenization of the system to a particular state, and pure dephasing were simulated via a collision model with identical uncorrelated environment particles in [34-36]. Even if environment particles are uncorrelated originally, they become partially correlated (entangled) with the system during collisions, so such an environment exhibits memory effects for further systems interacting with it [37-40]. Moreover, environment particles may be initially correlated (quantumly or classically) due to interactions between each other as it takes place in solids and quantum gases, and such correlations may result in non-Markovian dynamics [12,41-44]. NonMarkovian effects also appear in collision models, where the system can interact with the same environment particle several times [45,46], or an environment particle impacted by a system collides with another environment particle, which later collides with the system [47-50]. The latter scheme is equivalent to a scenario where the quantum system in question is coherently coupled to an auxiliary system interacting with the Markovian bath via collisions [51,52]. Collision models adequately describe a particle in semiquantal spin gases [53,54], a micromaser [55], a two-level system that interacts with spatiotemporal modes passing through it only once [56], and more complex systems with involved interaction graphs [57-59], as well as experiments with an engineered environment in nuclear magnetic resonance [12] and in photonic systems [11,60,61]. Collision models were also exploited in the microscopic description of Landauer's principle [62].

In the appropriate continuous limit of infinitesimal interaction time $\tau \rightarrow 0$, the collision model describes a smooth dynamical map $\Phi_{t}[57,63]$. Even if we consider simple interaction graphs, when the system interacts with each environment particle only once, collision models successfully simulate dynamical processes $\Phi_{t}$ with different divisibility properties [41]. So we resort to a collision model with generally correlated environment particles, with the correlations being attributed to prior interactions among environment constituents.

The second goal of this paper is to demonstrate that the divisibility property of the dynamical map $\Phi_{t}$ is closely related with the collision model describing it. Clearly, any $\mathrm{CP}$ divisible dynamics can be obtained with uncorrelated (factorized) environment states. We fully characterize ultimate CP divisible Pauli dynamical semigroups and corresponding collision models. CP indivisible dynamics necessarily involves correlations among environment particles.

Surprisingly, a convex sum $p_{1} \Phi_{t}^{(1)}+p_{2} \Phi_{t}^{(2)}+\cdots$ of CP divisible processes $\Phi_{t}^{(1)}, \Phi_{t}^{(1)}, \ldots$ can exhibit eternal CP indivisibility; for instance, this takes place for the convex sum of two dephasing dynamical maps [24,25]. We provide families of eternal CP indivisible processes and construct a collision model with correlated environment, which simulates them.

In contrast to a convex sum of dynamical maps, a conical (weighted) combination $\alpha \mathcal{L}_{t}^{(1)}+\beta \mathcal{L}_{t}^{(2)}$ of time-dependent generators $\mathcal{L}_{t}^{(1)}$ and $\mathcal{L}_{t}^{(2)}$ does not necessarily represent a valid generator $[64,65]$ unless master equations $\frac{\partial \varrho}{\partial t}=\mathcal{L}_{t}^{(1)}[\varrho]$ and $\frac{\partial \varrho}{\partial t}=\mathcal{L}_{t}^{(2)}[\varrho]$ both define $\mathrm{CP}$ divisible processes. When the latter condition is fulfilled, we demonstrate a collision model realizing the master equation $\frac{\partial \varrho}{\partial t}=\alpha \mathcal{L}_{t}^{(1)}[\varrho]+\beta \mathcal{L}_{t}^{(2)}[\varrho]$ for arbitrary non-negative weight coefficients $\alpha$ and $\beta$.

The paper is organized as follows. In Sec. II, we review divisibility properties of Pauli dynamical maps in pictorial representation. In Sec. III, ultimate CP divisible semigroups are studied. In Sec. IV, we provide a general collision model for ultimate CP divisible Pauli processes. In Sec. V, we demonstrate collision models that realize multiplicativity and additivity of time-local generators for $\mathrm{CP}$ divisible processes. In Sec. VI, we construct a correlated environment which leads to a mixture of CP divisible processes. In Sec. VII, a two-parameter family of eternal CP indivisible Pauli maps is presented. In Secs. VIII and IX, we review $\mathrm{P}$ divisible and $\mathrm{P}$ indivisible processes, respectively, as well as the physics of underlying collision models. In Sec. IX D, we provide a constructive collision model for an arbitrary Pauli dynamical map $\Phi_{t}$. In Sec. IXE, we discuss dynamical maps which continuously shrink the volume of accessible states, but are not $\mathrm{P}$ divisible. In Sec. $\mathrm{X}$, brief conclusions are given.

\section{DIVISIBILITY OF PAULI MAPS IN PICTORIAL REPRESENTATION}

A trajectory $\lambda(t)$ becomes particularly visual for Pauli qubit processes $\Phi_{t}: \mathcal{B}\left(\mathcal{H}_{2}\right) \mapsto \mathcal{B}\left(\mathcal{H}_{2}\right)$ that are characterized by three real parameters $\lambda_{1}(t), \lambda_{2}(t), \lambda_{3}(t)$ as follows:

$$
\Phi_{t}[\varrho]=\frac{1}{2}\left(\operatorname{tr}[\varrho] I+\sum_{j=1}^{3} \lambda_{j}(t) \operatorname{tr}\left[\sigma_{j} \varrho\right] \sigma_{j}\right)
$$

where $\sigma_{1}, \sigma_{2}, \sigma_{3}$ is a conventional set of Pauli operators. The map $\Phi_{t}$ is known to be positive if $-1 \leqslant \lambda_{1}(t), \lambda_{2}(t), \lambda_{3}(t) \leqslant$ 1 (cube in the parameter space) and completely positive if $1 \pm \lambda_{3}(t) \geqslant\left|\lambda_{1}(t) \pm \lambda_{2}(t)\right|$ (tetrahedron in the parameter space) $[66,67]$. In the case of a general physical evolution $\Phi_{t}$ with initially factorized system and environment, the trajectory 
(a)

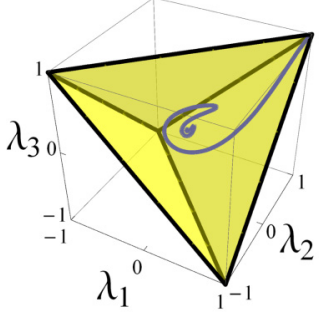

(c)

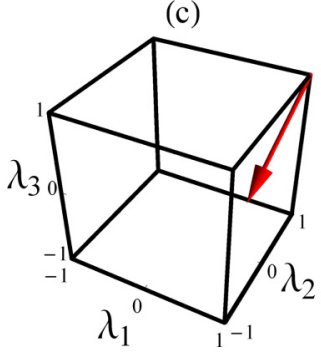

(b)

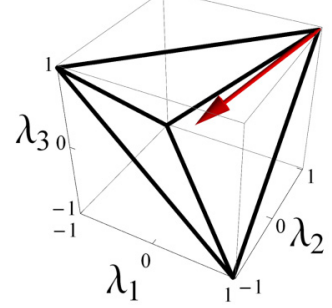

(d)

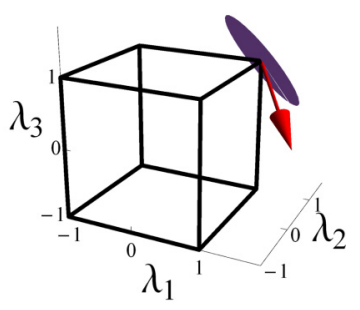

FIG. 1. (a) Physical Pauli processes correspond to curves inside the set of completely positive maps. The direction of the vector $\boldsymbol{\kappa}$ given by Eq. (6) defines the properties of Pauli dynamical maps: (b) CP divisibility, (c) P divisibility, and (d) monotonic shrink of the volume of accessible states.

$\lambda(t)$ can be an arbitrary smooth curve inside the tetrahedron $1 \pm \lambda_{3}(t) \geqslant\left|\lambda_{1}(t) \pm \lambda_{2}(t)\right|$ [see Fig. 1(a)].

Suppose the map $\Phi_{t}$ is invertible and $s$ tends to zero; then,

$$
\begin{aligned}
\Phi_{t+s}[\varrho(0)] & =\Phi_{t}[\varrho(0)]+s \dot{\Phi}_{t}[\varrho(0)] \\
& =\varrho(t)+s \dot{\Phi}_{t} \circ \Phi_{t}^{-1}[\varrho(t)]=\Theta_{t, t+s}[\varrho(t)],
\end{aligned}
$$

where $\dot{\Phi}_{t}=\frac{\partial}{\partial t} \Phi_{t}$. From Eq. (3), it follows that

$$
\Theta_{t, t+s}=\operatorname{Id}+s \dot{\Phi}_{t} \circ \Phi_{t}^{-1}
$$

as $s \rightarrow 0$.

The map $\dot{\Phi}_{t} \circ \Phi_{t}^{-1}$ defines a direction in the parameter space in which the process progresses. Using the explicit form of Eq. (2), we get

$$
\dot{\Phi}_{t} \circ \Phi_{t}^{-1}[X]=\frac{1}{2} \sum_{j=1}^{3} \frac{\dot{\lambda}_{j}(t)}{\lambda_{j}(t)} \operatorname{tr}\left[\sigma_{j} X\right] \sigma_{j},
$$

which identifies the vector

$$
\kappa(t)=\left(\frac{\dot{\lambda}_{1}(t)}{\lambda_{1}(t)}, \frac{\dot{\lambda}_{2}(t)}{\lambda_{2}(t)}, \frac{\dot{\lambda}_{3}(t)}{\lambda_{3}(t)}\right)
$$

representing the dynamical map in the parameter space of qubit unital channels. Let us stress that $\kappa(t)$ is not a tangent line to the trajectory $\lambda(t)$. Such vector $\kappa(t)$ can be drawn at any time moment $t$ for a sufficiently smooth trajectory $\lambda(t)$ in the parameter space, making the divisibility property more apparent.

In particular, if $s \rightarrow 0$, then the map $\Theta_{t, t+s}$ is completely positive if and only if the vector $\kappa(t)$ drawn from the corner $(1,1,1)$ of the parameter space points inside the tetrahedron of completely positive maps in Fig. 1(b), i.e., the scalar products of $\kappa(t)$ with vectors $(-1,1,1),(1,-1,1)$, and $(1,1,-1)$ are all nonpositive:

$$
\begin{gathered}
-\kappa_{1}(t)+\kappa_{2}(t)+\kappa_{3}(t) \leqslant 0, \\
\kappa_{1}(t)-\kappa_{2}(t)+\kappa_{3}(t) \leqslant 0 \\
\kappa_{1}(t)+\kappa_{2}(t)-\kappa_{3}(t) \leqslant 0 .
\end{gathered}
$$

Analogously, if $s \rightarrow 0$, then the map $\Theta_{t, t+s}$ is positive if and only if the vector $\kappa(t)$ drawn from the corner $(1,1,1)$ of the parameter space points inside the cube of positive maps in Fig. 1(c), i.e., the scalar products of $\kappa(t)$ with vectors $(1,0,0)$, $(0,1,0)$, and $(0,0,1)$ are all nonpositive:

$$
\kappa_{1}(t) \leqslant 0, \quad \kappa_{2}(t) \leqslant 0, \quad \kappa_{3}(t) \leqslant 0 .
$$

For the uniform measure of qubit states inside the Bloch ball (metric induced by Hilbert-Schmidt distance [67]), the volume of accessible states for the Pauli map is $V(t)=$ $\left|\lambda_{1}(t) \lambda_{2}(t) \lambda_{3}(t)\right|$. The map $\Theta_{t, t+s}$ shrinks the volume of accessible states if and only if $\prod_{i=1}^{3}\left(1+s \kappa_{i}(t)\right) \leqslant 1$, which in the limit $s \rightarrow 0$ transforms into requirement

$$
\kappa_{1}(t)+\kappa_{2}(t)+\kappa_{3}(t) \leqslant 0 .
$$

Here we have taken into account that if $\kappa_{1}+\kappa_{2}+\kappa_{3}=0$ and at least one $\kappa_{i} \neq 0$, then $\kappa_{1} \kappa_{2}+\kappa_{2} \kappa_{3}+\kappa_{3} \kappa_{1}=-\frac{1}{2}\left(\kappa_{1}^{2}+\right.$ $\left.\kappa_{2}^{2}+\kappa_{3}^{2}\right)<0$, which implies $\prod_{i=1}^{3}\left(1+s \kappa_{i}\right)<1$. Geometrically, the vector $\kappa(t)$ has nonpositive scalar product with the vector $(1,1,1)$, i.e., the vector $\kappa(t)$ drawn from the corner $(1,1,1)$ in parameter space points to a specific half space separated by the plane $\lambda_{1}+\lambda_{2}+\lambda_{3}=3$ [see Fig. 1(d)].

\section{ULTIMATE CP DIVISIBILITY OF SEMIGROUP DYNAMICS}

Consider a semigroup dynamics $\Phi_{t}=e^{\mathcal{L} t}$, where $\mathcal{L}$ : $\mathcal{B}\left(\mathcal{H}_{2}\right) \mapsto \mathcal{B}\left(\mathcal{H}_{2}\right)$ is a time-independent generating map of the form $[68,69]$

$$
\mathcal{L}[\varrho]=-i[H, \varrho]+\sum_{k} \gamma_{k}\left(A_{k} \varrho A_{k}^{\dagger}-\frac{1}{2}\left\{\varrho, A_{k}^{\dagger} A_{k}\right\}\right),
$$

where $H$ is Hermitian and $\gamma_{k} \geqslant 0$. It follows that for semigroup dynamics, the identity $\Phi_{t+s}=\Phi_{t} \circ \Phi_{s}$ holds for all $t, s \geqslant 0$. Consequently, $\Theta_{t, t+s}=\Phi_{s}=e^{\mathcal{L} s}$, and hence, the semigroup dynamics is always CP divisible.

The time evolution of the density operator is given by equation

$$
\frac{\partial \varrho}{\partial t}=\mathcal{L}[\varrho]
$$

Consider now an infinitesimal perturbations of Eq. (13),

$$
\frac{\partial \varrho}{\partial t}=\left(\mathcal{L}+\delta \mathcal{L}_{t}\right)[\varrho],
$$

where $\delta \mathcal{L}_{0}=0$. The term $\delta \mathcal{L}_{t}$ describes an infinitely differentiable deviation from dynamics (13) and can be attributed to, e.g., a slightly modified environment or a fluctuating interaction between system and environment. By definition, we say that a semigroup dynamics is ultimate $C P$ divisible if it becomes CP indivisible under some perturbation $\delta \mathcal{L}$. 
For qubit unital semigroup processes (2), we have $\lambda_{j}(t)=$ $e^{-\Gamma_{j} t}$ and, consequently, the vector $\kappa(t)=-\Gamma$ is time independent and we used $\boldsymbol{\Gamma}=\left(\Gamma_{1}, \Gamma_{2}, \Gamma_{3}\right)$. By definition, the perturbations $\delta \mathcal{L}_{t}$ are introducing only minor changes and the deviated vector $\boldsymbol{\kappa}+\delta \boldsymbol{\kappa}$ would satisfy Eqs. (7)-(9) whenever these inequalities for the unperturbed case $\boldsymbol{\kappa}$ are strict. It turns out that qubit unital semigroup dynamics can be ultimately CP only if $\kappa_{i}+\kappa_{j}-\kappa_{k}=0$ for some permutation of indexes $i, j, k \in\{1,2,3\}$. In fact, in such case, there exists an infinitesimal perturbation $\delta \mathcal{L}_{t}$ resulting in a dynamical map $\Phi_{t}+\delta \Phi_{t}$ violating Eqs. (7)-(9). Taking into account the definition of $\kappa$, we find that the condition $\kappa_{i}+\kappa_{j}-\kappa_{k}=0$ translates into differential equation $\frac{d}{d t} \ln \left(\lambda_{i} \lambda_{j}\right)=\frac{d}{d t} \ln \left(\lambda_{k}\right)$ with the solution $\lambda_{i}(t) \lambda_{j}(t)=c \lambda_{k}(t)$, where the constant $c$ can be found from the initial condition $\lambda_{1}(0)=\lambda_{2}(0)=\lambda_{3}(0)=1$. In conclusion, $c=1$ and ultimate $\mathrm{CP}$ divisible unital processes satisfy the identity

$$
\lambda_{i}(t) \lambda_{j}(t)=\lambda_{k}(t) .
$$

A general qubit unital semigroup evolution (up to unitary freedom) takes the form

$$
\mathcal{L}[\varrho]=-\frac{1}{2} \sum_{j=1}^{3} \Gamma_{j} \operatorname{tr}\left[\sigma_{j} \varrho\right] \sigma_{j}=\frac{1}{2} \sum_{j=1}^{3} \gamma_{j}\left(\sigma_{j} \varrho \sigma_{j}-\varrho\right),
$$

where $\Gamma_{j}^{-1}$ are experimentally measurable time scales of decoherence processes and $\gamma_{j}$ are dissipator rates given by the formula

$$
\left(\begin{array}{l}
\gamma_{1} \\
\gamma_{2} \\
\gamma_{3}
\end{array}\right)=\frac{1}{2}\left(\begin{array}{ccc}
-1 & 1 & 1 \\
1 & -1 & 1 \\
1 & 1 & -1
\end{array}\right)\left(\begin{array}{l}
\Gamma_{1} \\
\Gamma_{2} \\
\Gamma_{3}
\end{array}\right)
$$

The conditions of ultimate CP divisibility on $\boldsymbol{\kappa}$ imply $\Gamma_{i}+$ $\Gamma_{j}-\Gamma_{k}=\gamma_{k}=0$ (for some permutation of indexes $i, j, k$ ). It follows that the generator for the ultimate CP divisible Pauli semigroup contains at most two terms,

$$
\mathcal{L}[\varrho]=\frac{\gamma_{i}}{2}\left(\sigma_{i} \varrho \sigma_{i}-\varrho\right)+\frac{\gamma_{j}}{2}\left(\sigma_{j} \varrho \sigma_{j}-\varrho\right),
$$

and the trajectory in the parameter space is $\lambda_{i}=e^{-\gamma_{j} t}, \lambda_{j}=$ $e^{-\gamma_{i} t}, \lambda_{k}=e^{-\left(\gamma_{i}+\gamma_{j}\right) t}$. The class of time evolutions for ultimate $\mathrm{CP}$ divisible Pauli semigroups is illustrated in Fig. 2. In the next section, we will provide a physical realization of the generator (18).

Physical examples of ultimate $\mathrm{CP}$ divisible processes include the following:

(i) pure phase damping process, when $\lambda_{i}(t)=1, \lambda_{j}(t)=$ $\lambda_{k}(t)=e^{-\Gamma t}$ and corresponding to the choice of dissipation rates $\gamma_{j}=\gamma_{k}=0$ (green lines in Fig. 2);

(ii) generalized amplitude damping process with hightemperature environment ([70], Sec. 8.3.5), i.e., a spontaneous decay with equal probabilities of energy absorption and emission, when $\lambda_{i}(t)=\lambda_{j}(t)=e^{-\Gamma t}$ and $\lambda_{k}(t)=e^{-2 \Gamma t}$ in a Markov approximation ([32], Sec. 10.1),

$$
\mathcal{L}[\varrho]=\Gamma\left(\sigma_{+} \varrho \sigma_{-}+\sigma_{-} \varrho \sigma_{+}-\varrho\right),
$$

where $\sigma_{ \pm}=\frac{1}{2}\left(\sigma_{i} \pm i \sigma_{j}\right)$ are excitation creation and annihilation operators. This process is illustrated as the bottom red line in Fig. 2 and corresponds to the choice of dissipation rates $\gamma_{i}=\gamma_{j}$ and $\gamma_{k}=0$.

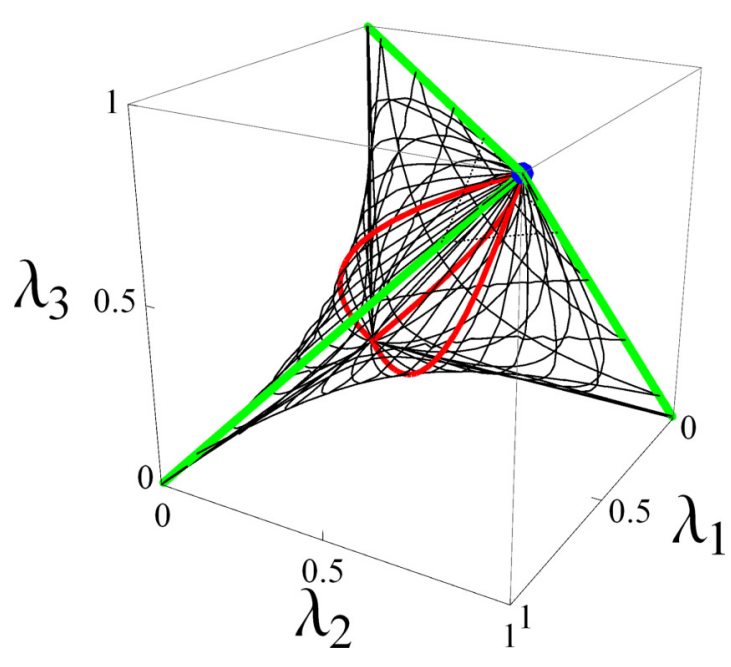

FIG. 2. Ultimate CP divisible semigroups among Pauli dynamical maps. Green (thick straight) lines correspond to pure dephasing processes. Red (thick curved) lines correspond to generalized amplitude damping processes with infinite temperature of the environment.

Any Pauli channel $\Phi$ with parameters $\lambda_{1}, \lambda_{2}, \lambda_{3}$ inside the body determined by ultimate CP divisible processes in Fig. 2 can be obtained as a result of some semigroup dynamics with a particular generator $\mathcal{L}$ and time period $t$, i.e., $\Phi=e^{\mathcal{L} t}$. Moreover, even if the parameters of the generator $\mathcal{L}$ in Eq. (16) are time dependent but with positive decoherence rates (so-called time-dependent Markovian dynamics [16]), then achievable channels $\Phi_{t}$ still belong to the body in Fig. 2. Assigning equal weights to all Pauli channels, the fraction of semigroupachievable quantum channels equals $\frac{V_{\text {body }}}{V_{\text {tetrahedron }}}=\frac{3}{32}=9.375 \%$, which is comparable with the numerical estimations of general (nonunital) qubit semigroup-achievable channels (2\%) and general (nonunital) qubit channels achievable by timedependent Markovian dynamics (17\%); see Ref. [16].

\section{COLLISION MODELS OF ULTIMATE CP DIVISIBLE SEMIGROUPS}

Physically, the evolution $\frac{\partial}{\partial t} \varrho=\mathcal{L}[\varrho]$ with dissipator (18) is achievable as a result of sequential interactions of the system qubit with environment qubits (collision model, Fig. 3). Let all environment qubits be in the same state $\xi=\frac{1}{2} I$ (Fig. 4). The system qubit and the $n$th environment qubit interact pairwise during the time period $\tau$, with the interaction Hamiltonian

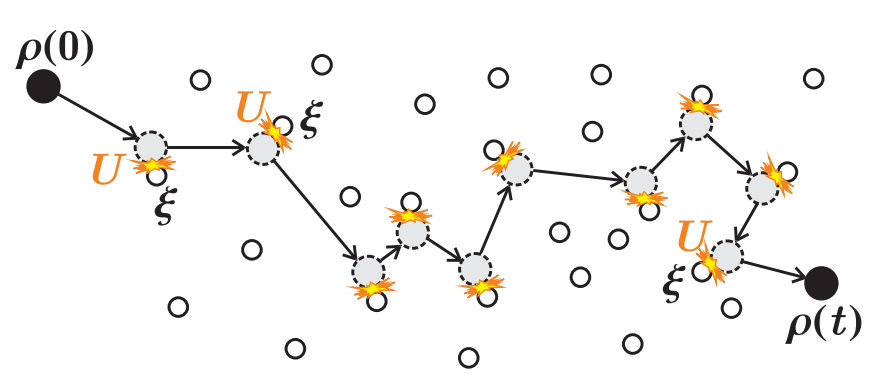

FIG. 3. Physics of collision model. 


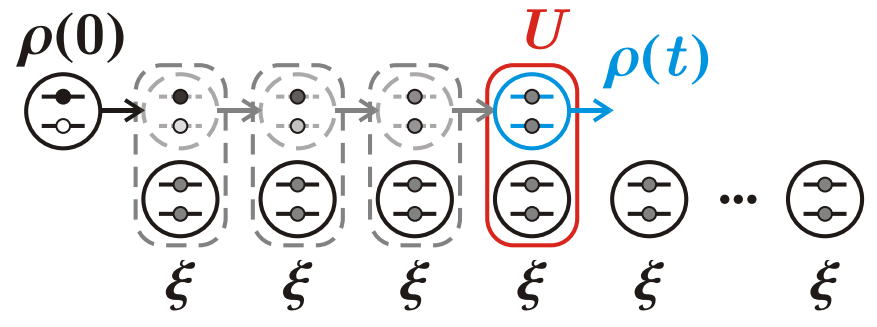

FIG. 4. Collision model for Pauli dynamical maps with ultimate $\mathrm{CP}$ divisible semigroup property.

being

$$
H_{\mathrm{int}}=\frac{1}{2}\left(g_{1} \sigma_{x} \otimes \sigma_{x}+g_{2} \sigma_{y} \otimes \sigma_{y}\right) .
$$

The system qubit and the $n$th environment qubit experience the unitary transformation

$$
\begin{aligned}
U_{\tau}= & \exp \left(-i H_{\mathrm{int}} \tau\right) \\
= & \cos \frac{g_{1} \tau}{2} \cos \frac{g_{2} \tau}{2} I \otimes I-i \sin \frac{g_{1} \tau}{2} \cos \frac{g_{2} \tau}{2} \sigma_{x} \otimes \sigma_{x} \\
& -i \cos \frac{g_{1} \tau}{2} \sin \frac{g_{2} \tau}{2} \sigma_{y} \otimes \sigma_{y} \\
& +\sin \frac{g_{1} \tau}{2} \sin \frac{g_{2} \tau}{2} \sigma_{z} \otimes \sigma_{z} .
\end{aligned}
$$

As a result of such an interaction, the system state $\varrho$ transforms as follows:

$$
\varrho \longrightarrow \Phi_{\tau}[\varrho]=\operatorname{tr}_{n}\left\{U_{\tau}\left(\varrho \otimes \frac{1}{2} I\right) U_{\tau}^{\dagger}\right\}
$$

where $\operatorname{tr}_{n}$ denotes the partial trace over the $n$th environment qubit. Some algebra yields the single interaction elementary map $\Phi_{\tau}$, which is unital, does not depend on $n$, and reads

$$
\begin{aligned}
\Phi_{\tau}[\varrho]= & \frac{1}{2}\left(\operatorname{tr}[\varrho] I+\cos \left(g_{2} \tau\right) \operatorname{tr}\left[\sigma_{x} \varrho\right] \sigma_{x}+\cos \left(g_{1} \tau\right) \operatorname{tr}\left[\sigma_{y} \varrho\right] \sigma_{y}\right. \\
& \left.+\cos \left(g_{1} \tau\right) \cos \left(g_{2} \tau\right) \operatorname{tr}\left[\sigma_{z} \varrho\right] \sigma_{z}\right) .
\end{aligned}
$$

Since the system qubit always interacts with a fresh environmental particle, after $\frac{t}{\tau}$ interactions we get the dynamical map

$$
\Phi_{t}=\left(\Phi_{\tau}\right)^{t / \tau}
$$

with parameters $\lambda_{1}(t)=\left[\cos \left(g_{2} \tau\right)\right]^{t / \tau}, \lambda_{2}(t)=\left[\cos \left(g_{1} \tau\right)\right]^{t / \tau}$, and $\lambda_{3}(t)=\lambda_{1}(t) \lambda_{2}(t)$. In the stroboscopic limit $[39,57,71]$ $\tau \rightarrow 0, g_{1}^{2} \tau \rightarrow 2 \gamma_{1}, g_{2}^{2} \tau \rightarrow 2 \gamma_{2}$, we get the continuous dynamics $\lambda_{1}(t)=e^{-\gamma_{2} t}, \lambda_{2}(t)=e^{-\gamma_{1} t}$, and $\lambda_{3}(t)=e^{-\left(\gamma_{1}+\gamma_{2}\right) t}$. Thus, parameters $\lambda_{1}(t), \lambda_{2}(t)$, and $\lambda_{3}(t)$ satisfy condition (15) and the induced dynamics is ultimate CP divisible.

This proves that ultimate $\mathrm{CP}$ divisible dynamics with the dissipator (18) can be realized in the stroboscopic limit of the collision model with the elementary pairwise Hamiltonian $H=g_{i} \sigma_{i} \otimes \sigma_{i}+g_{j} \sigma_{j} \otimes \sigma_{j}$, where the coefficients $g_{i}$ and $g_{j}$ satisfy $\frac{g_{i}}{g_{j}}=\sqrt{\frac{\gamma_{i}}{\gamma_{j}}}$. Trajectories of ultimate CP divisible Pauli semigroups are depicted in Fig. 2.

\section{MULTIPLICATIVITY AND ADDITIVITY OF GENERATORS IN COLLISION MODELS}

Any CP divisible process $\Phi_{t}$ can be realized stroboscopically via a collision model with the arbitrary chosen precision. In fact, since $\Theta_{t, t+s}$ is a valid dynamical map for all $t$ and $s$, its dilation (unitary operator $V_{t, t+s}$ and environment state $\xi_{t, t+s}$ ) is continuous with respect to $t$ and $s$ [72]. Fixing $s=\tau$, we get a sequence of environment states,

$$
\xi_{0, \tau}, \xi_{\tau, 2 \tau}, \ldots, \xi_{(n-1) \tau, n \tau}, \ldots
$$

and a sequence of unitary operators acting on the system and $n$th environment particle,

$$
V_{0, \tau}, V_{\tau, 2 \tau}, \ldots, V_{(n-1) \tau, n \tau}, \ldots,
$$

such that the dynamics $\Phi_{t}[\varrho]$ coincides with the simulation $\Phi_{n \tau}^{\operatorname{sim}}[\varrho]=\operatorname{tr}_{\mathrm{env}}\left[V_{(n-1) \tau, n \tau} \cdots V_{\tau, 2 \tau} V_{0, \tau}\left(\varrho \otimes \xi_{0, \tau} \otimes\right.\right.$ $\left.\left.\xi_{\tau, 2 \tau} \otimes \cdots \otimes \xi_{(n-1) \tau, n \tau}\right) V_{0, \tau}^{\dagger} V_{\tau, 2 \tau}^{\dagger} \cdots V_{(n-1) \tau, n \tau}^{\dagger}\right]$ at time moments $t=n \tau$. Thus, there exists a collision model with factorized environment which simulates master equation $\frac{\partial \varrho}{\partial t}=$ $\mathcal{L}_{t}[\varrho]$ for the generator $\mathcal{L}_{t}=\dot{\Phi}_{t} \circ \Phi_{t}^{-1}$ if $\Phi_{t}$ is CP divisible.

Analogously, if we replace the generator $\mathcal{L}_{t}$ by $\alpha \mathcal{L}_{t}$ with some positive $\alpha$, then the resulting evolution is still CP divisible and can be realized stroboscopically at the same time moments $t=n \tau$ (each collision increments time by $\tau$ ) with a modified sequence of environment states,

$$
\xi_{0, \alpha \tau}, \xi_{\tau,(1+\alpha) \tau}, \ldots, \xi_{(n-1) \tau,(n-1+\alpha) \tau}, \ldots,
$$

and a sequence of unitary operators acting on the system and $n$th environment particle,

$$
V_{0, \alpha \tau}, V_{\tau,(1+\alpha) \tau}, \ldots, V_{(n-1) \tau,(n-1+\alpha) \tau}, \ldots
$$

Note that such an apparent construction of a collision model for multiplicative generator $\alpha \mathcal{L}_{t}$ is valid only if the original process $\Phi_{t}$ is CP divisible. If this is not the case, the modified master equation $\frac{\partial \varrho}{\partial t}=\alpha \mathcal{L}_{t}[\varrho]$ may lead to nonphysical solutions, with the example being presented in Ref. [65].

Consider two CP divisible processes $\Phi_{t}^{(1)}$ and $\Phi_{t}^{(2)}$ defined via master equations $\frac{\partial \varrho}{\partial t}=\mathcal{L}_{t}^{(1)}[\varrho]$ and $\frac{\partial \varrho}{\partial t}=\mathcal{L}_{t}^{(2)}[\varrho]$, respectively. Each dynamical map $\Phi_{t}^{(i)}$ can be simulated stroboscopically with a sequence of environment states $\left\{\xi_{(n-1) \tau, n \tau}^{(i)}\right\}$ and unitary operators $\left\{V_{(n-1) \tau, n \tau}^{(i)}\right\}, i=1,2$. If the system interacts during time $\tau$ alternatively with particles from the first and second sequences, i.e., with particles from the first environment at odd collisions and with particles from the second environment at even collisions (Fig. 5), then the resulting dynamics simulates the master equation $\frac{\partial \varrho}{\partial t}=\frac{1}{2}\left(\mathcal{L}_{t}^{(1)}+\mathcal{L}_{t}^{(2)}\right)[\varrho]$ at times $t=2 n \tau$. In a more general physical situation, when the system interacts independently with two types of environments, the effective generator reads $p_{1} \mathcal{L}_{t}^{(1)}+p_{2} \mathcal{L}_{t}^{(2)}$, where $p_{1}$ and $p_{2}$ are the probabilities of encountering a particle from the first and second environment, respectively. Therefore, additivity of generators can be realized in a stroboscopic model if those generators lead to CP divisible dynamics. When the latter condition is violated, the addition of generators may also lead to nonphysical solutions [64]. 


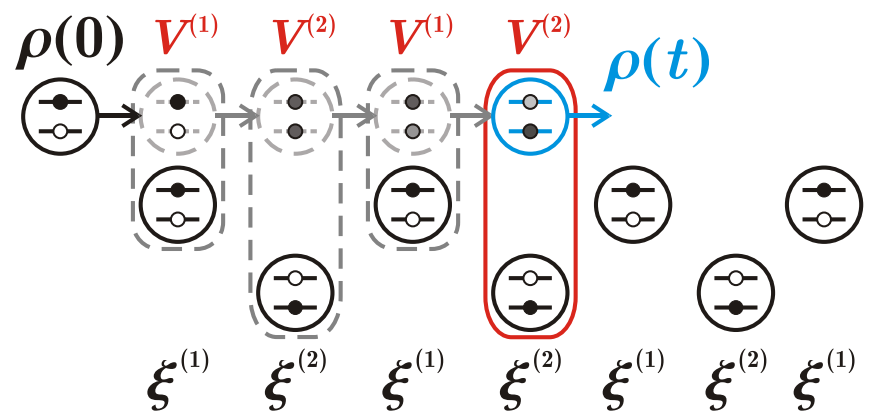

FIG. 5. Simulation of generator $\frac{1}{2}\left(\mathcal{L}_{t}^{(1)}+\mathcal{L}_{t}^{(2)}\right)$ for CP divisible dynamical maps governed by master equations $\frac{\partial \varrho}{\partial t}=\mathcal{L}_{t}^{(1)}[\varrho]$ and $\frac{\partial \varrho}{\partial t}=$ $\mathcal{L}_{t}^{(2)}[\varrho]$.

\section{MIXTURES OF CP DIVISIBLE PROCESSES}

Consider a dynamical map which is a mixture of $\mathrm{CP}$ divisible processes,

$$
\Phi_{t}=\sum_{m=1}^{M} p_{m} \Phi_{t}^{(m)}
$$

where $\left\{p_{m}\right\}$ are the probabilities with which CP divisible dynamical maps $\left\{\Phi_{t}^{(m)}\right\}$ contribute to the map $\Phi_{t}, p_{m} \geqslant 0$ and $\sum_{m=1}^{M} p_{m}=1$. Note that this situation is substantially different from the weighted sum of generators since $\mathcal{L}_{t}=\dot{\Phi}_{t} \circ \Phi_{t}^{-1} \neq$ $\sum_{m=1}^{M} w_{m} \dot{\Phi}_{t}^{(m)} \circ\left(\Phi_{t}^{(m)}\right)^{-1}=\sum_{m=1}^{M} w_{m} \mathcal{L}_{t}^{(m)}$, in general.

Surprisingly, even if all the processes $\Phi_{t}^{(m)}$ are CP divisible, $\Phi_{t}$ can still be CP indivisible. The prominent example is the mixture

$$
\Phi_{t}^{\text {mix }}=p_{1} e^{\mathcal{L}_{1} t}+p_{2} e^{\mathcal{L}_{2} t}+p_{3} e^{\mathcal{L}_{3} t}
$$

of purely dephasing maps $e^{\mathcal{L}_{i} t}$ with $\mathcal{L}_{i}[\varrho]=\gamma\left(\sigma_{i} \varrho \sigma_{i}-\varrho\right)$. In Ref. [24], the region of simplex $\left(p_{1}, p_{2}, p_{3}\right)$ is found, for which $\Phi_{t}^{\text {mix }}$ is not CP divisible for all $t>t_{*}$. If only one of the probabilities $p_{1}, p_{2}, p_{3}$ equals zero, then $\Phi_{t}^{\text {mix }}$ is eternal CP indivisible.

In what follows, we will design a collision model simulation of a general mixture $\Phi_{t}=\sum_{m=1}^{M} p_{m} \Phi_{t}^{(m)}$ of CP divisible dynamical maps. Suppose each $\Phi_{t}^{(m)}$ is realized by a collision model with environment states $\xi_{1}^{(m)}, \xi_{2}^{(m)}, \ldots, \xi_{n}^{(m)}$ and elementary unitary transformations $U_{1}^{(m)}=\exp \left(-i H_{1}^{(m)} \tau\right), U_{2}^{(m)}=$ $\exp \left(-i H_{2}^{(m)} \tau\right), \ldots, U_{n}^{(m)}=\exp \left(-i H_{n}^{(m)} \tau\right)$. The whole environment of the $m$ th process reads $\Xi^{(m)}=\xi_{1}^{(m)} \otimes \xi_{2}^{(m)} \otimes \cdots \otimes$ $\xi_{n}^{(m)}=\bigotimes_{k=1}^{n} \xi_{k}^{(m)}$. In a probabilistic sense, the mixture can be realized as a mixture of collision models for each individual $\Phi_{t}^{(m)}$; however, such implementation is not "operationally faithful" because in each run of the experiment a randomly chosen but different CP divisible process $\Phi_{t}^{(m)}$ is realized. We will present an alternative realization of such mixtures and design a collision model with a correlated state of the environment implementing the desired mixture in each individual run of the experiment.
In particular, consider the following initial state of the environment:

$$
\begin{aligned}
& \Xi=\bigoplus_{m=1}^{M} p_{m} \Xi^{(m)}=\left(\begin{array}{llll}
p_{1} \Xi^{(1)} & & & \\
& p_{2} \Xi^{(2)} & & \\
& & \ddots & \\
& & & p_{M} \Xi^{(M)}
\end{array}\right)
\end{aligned}
$$

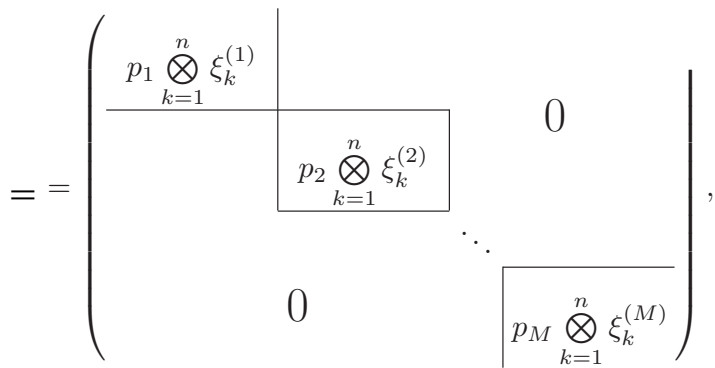

which does not have the tensor product structure with respect to collisions, i.e., $\Xi \neq \xi_{1} \otimes \xi_{2} \otimes \cdots \otimes \xi_{n}$. Let us note that this state is correlated, but not entangled. Also, note that the Hermitian operator $H_{k}^{(m)}$ is an interaction Hamiltonian between the system and the $k$ th particle of the $m$ th environment, so $H_{k}^{(m)}$ acts nontrivially on vectors in the subspace $\mathcal{H}_{\text {sys }} \otimes \mathcal{H}_{k}^{(m)}$ only. In other words, $H_{k}^{(m)}$ involves degrees of freedom of the system and the $k$ th particle of the $m$ th block of matrix (31). Consequently, $H_{k}^{(m)} H_{k}^{\left(m^{\prime}\right)}=0$ if $m \neq m^{\prime}$. The combined Hamiltonian $H_{k}=\sum_{m=1}^{M} H_{k}^{(m)}$ generates the unitary evolution operator,

$$
\begin{aligned}
U_{k}= & \exp \left(-i H_{k} \tau\right)=\sum_{l=0}^{\infty} \frac{(-i \tau)^{l}}{l !}\left(H_{k}\right)^{l}=\sum_{l=0}^{\infty} \frac{(-i \tau)^{l}}{l !} \\
& \times \sum_{m=1}^{M}\left(H_{k}^{(m)}\right)^{l}=\sum_{m=1}^{M} \exp \left(-i H_{k}^{(m)} \tau\right)=\sum_{m=1}^{M} U_{k}^{(m)},
\end{aligned}
$$

where the support of $U_{k}^{(m)}=\exp \left(-i H_{k}^{(m)} \tau\right)$ is $\mathcal{H}_{\text {sys }} \otimes \mathcal{H}_{k}^{(m)}$, so $U_{k}^{(m)} U_{k}^{\left(m^{\prime}\right)}=0$ if $m \neq m^{\prime}$. The sequence of $n$ collisions results in the evolution operator

$$
U_{n} \cdots U_{2} U_{1}=\sum_{m=1}^{M} U_{n}^{(m)} \cdots U_{2}^{(m)} U_{1}^{(m)},
$$

where $U_{n}^{(m)} \cdots U_{2}^{(m)} U_{1}^{(m)}$ does not vanish on vectors involving the system and the $m$ th block of matrix (31).

The dynamical map after $n$ collisions reads

$$
\begin{aligned}
\Phi[\varrho] & =\operatorname{tr}_{\mathrm{env}}\left[U_{n} \cdots U_{2} U_{1} \varrho \otimes \Xi U_{1}^{\dagger} U_{2}^{\dagger} \cdots U_{n}^{\dagger}\right] \\
& =\sum_{m=1}^{M} p_{m} \operatorname{tr}_{\mathrm{env}}\left[U_{n}^{(m)} \cdots U_{1}^{(m)} \varrho \otimes \Xi^{(m)} U_{1}^{(m) \dagger} \cdots U_{n}^{(m) \dagger}\right] \\
& =\sum_{m=1}^{M} p_{m} \Phi^{(m)}[\varrho] .
\end{aligned}
$$

Therefore, the correlated environment (31) enables realization of the mixture of dynamical maps (29). 


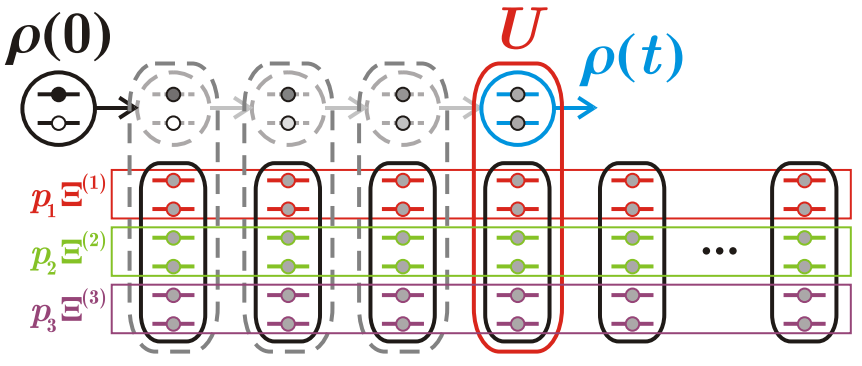

FIG. 6. Collision model with correlated environment, which realizes the deterministic mixture of $\mathrm{CP}$ divisible processes.

Example 1. Consider a mixture of pure dephasing qubit channels, given by Eq. (30), $M=3$. The deterministic collision model of such a dynamics is achieved with the environment composed of $n$ six-level systems (Fig. 6). The classically correlated state of $n$ environment particles is

$\Xi=\left(p_{1} \bigotimes_{k=1}^{n} \frac{1}{2} I^{(1)}\right) \bigoplus\left(p_{2} \bigotimes_{k=1}^{n} \frac{1}{2} I^{(2)}\right) \bigoplus\left(p_{3} \bigotimes_{k=1}^{n} \frac{1}{2} I^{(3)}\right)$,

which assigns probability $p_{1}\left(p_{2}, p_{3}\right)$ to the occurrence of collision with the first (second, third) pair of levels within the six-level system.

Elementary unitary transformations $U_{k}^{(m)}$ coincide for all collisions $k=1, \ldots, n$ and represent a generalization of a controlled-unitary operation, where the system is a controlled qubit, and the $m$ th qubit within the triple serves as a controlling qubit,

$$
U_{k}^{(m)}=e^{i g \tau \sigma_{m}} \otimes\left|0_{m}\right\rangle\left\langle 0_{m}\left|+e^{-i g \tau \sigma_{m}} \otimes\right| 1_{m}\right\rangle\left\langle 1_{m}\right| .
$$

In the stroboscopic limit [39,57,71], $\tau \rightarrow 0$ and $g^{2} \tau \rightarrow$ $2 \gamma$, Eq. (35) leads to the dynamical map $\Phi_{t}^{\text {mix }}=p_{1} e^{\mathcal{L}_{1} t}+$ $p_{2} e^{\mathcal{L}_{2} t}+p_{3} e^{\mathcal{L}_{3} t}$ with $\mathcal{L}_{i}[\varrho]=\gamma\left(\sigma_{i} \varrho \sigma_{i}-\varrho\right)$.

\section{ETERNAL CP INDIVISIBILITY}

Eternal CP indivisible dynamical maps $\Phi_{t}$ are those that are not CP divisible for any time $t>0$. It was shown recently that eternal $\mathrm{CP}$ indivisibility is quite a general property for approximate master equations describing spinboson systems [73]. Known examples of eternal CP indivisible Pauli dynamical maps include nontrivial convex combinations $p_{i} \Phi_{t}^{\mathrm{pd} i}+p_{j} \Phi_{t}^{\mathrm{pd} j}$ of pure dephasing processes $\Phi_{t}^{\mathrm{pd} i}$ and $\Phi_{t}^{\mathrm{pd} j}$ (in the basis of eigenstates of operators $\sigma_{i}$ and $\sigma_{j}$, respectively), $i, j=x, y, z, i \neq j[24,25]$. In what follows, we extend this one-parameter family (since $p_{i}+p_{j}=1$ ) to a wider class, namely, a two-parameter family of eternal CP indivisible maps. The underlying idea is to consider such smooth trajectories $\lambda(t)$ in the parameter space $\lambda_{1}, \lambda_{2}, \lambda_{3}$ that do not belong to the geometrical body in Fig. 2. These trajectories are beyond ultimate $\mathrm{CP}$ divisible processes, and as a result the $\boldsymbol{\kappa}$ vector always points beyond the tetrahedron in Fig. 1(b).

To start with, let us focus on three ultimate CP divisible semigroup processes:

(i) $\Phi_{t}^{(1)}=e^{\mathcal{L}^{(1)} t} \quad$ with the dissipator $\quad \mathcal{L}^{(1)}[\varrho]=$ $\frac{\gamma_{i}}{2}\left(\sigma_{i} \varrho \sigma_{i}-\varrho\right)+\frac{\gamma_{j}}{2}\left(\sigma_{j} \varrho \sigma_{j}-\varrho\right), \quad i \neq j, \quad \gamma_{i, j}>0, \quad$ which describes a "skewed" amplitude damping process towards a completely mixed state $\frac{1}{2} I$ via contact with a high-temperature environment;

(ii) $\Phi_{t}^{(2)}=e^{\mathcal{L}^{(2)} t} \quad$ with the dissipator $\quad \mathcal{L}^{(2)}[\varrho]=$ $\frac{\gamma_{i}}{2}\left(\sigma_{i} \varrho \sigma_{i}-\varrho\right)$, which is a pure phase damping process in the basis of eigenstates of $\sigma_{i}$; and

(iii) $\Phi_{t}^{(3)}=e^{\mathcal{L}^{(3)} t} \quad$ with the dissipator $\quad \mathcal{L}^{(3)}[\varrho]=$ $\frac{\gamma_{j}}{2}\left(\sigma_{j} \varrho \sigma_{j}-\varrho\right)$, which is a pure phase damping process in the basis of eigenstates of $\sigma_{j}$.

Let us demonstrate that any nontrivial mixture $\Phi_{t}=$ $p_{1} \Phi_{t}^{(1)}+p_{2} \Phi_{t}^{(2)}+p_{3} \Phi_{t}^{(3)}$ with $p_{1,2,3}>0$ is eternal CP indivisible. In fact, parameters of the unital map $\Phi_{t}$ read

$$
\begin{gathered}
\lambda_{i}(t)=\left(p_{1}+p_{3}\right) e^{-\gamma_{j} t}+p_{2}, \\
\lambda_{j}(t)=\left(p_{1}+p_{2}\right) e^{-\gamma_{i} t}+p_{3}, \\
\lambda_{k}(t)=p_{1} e^{-\left(\gamma_{i}+\gamma_{j}\right) t}+p_{2} e^{-\gamma_{i} t}+p_{3} e^{-\gamma_{j} t} .
\end{gathered}
$$

Calculation of the $\kappa$ vector yields

$$
\begin{gathered}
\kappa_{i}(t)=-\frac{\gamma_{j}\left(p_{1}+p_{3}\right)}{p_{1}+p_{3}+p_{2} e^{\gamma_{j} t}}, \\
\kappa_{j}(t)=-\frac{\gamma_{i}\left(p_{1}+p_{2}\right)}{p_{1}+p_{2}+p_{3} e^{\gamma_{i} t}}, \\
\kappa_{k}(t)=-\frac{\gamma_{i}\left(p_{1}+p_{2} e^{\gamma_{j} t}\right)+\gamma_{j}\left(p_{1}+p_{3} e^{\gamma_{i} t}\right)}{p_{1}+p_{2} e^{\gamma_{j} t}+p_{3} e^{\gamma_{i} t}} .
\end{gathered}
$$

Since the inequalities

$$
\begin{aligned}
& \frac{p_{1}+p_{2} e^{\gamma_{j} t}}{p_{1}+p_{2} e^{\gamma_{j} t}+p_{3} e^{\gamma_{i} t}}>\frac{p_{1}+p_{2}}{p_{1}+p_{2}+p_{3} e^{\gamma_{i} t}}, \\
& \frac{p_{1}+p_{3} e^{\gamma_{i} t}}{p_{1}+p_{2} e^{\gamma_{j} t}+p_{3} e^{\gamma_{i} t}}>\frac{p_{1}+p_{3}}{p_{1}+p_{3}+p_{2} e^{\gamma_{j} t}}
\end{aligned}
$$

hold true for all $t>0$, we conclude that $\kappa_{i}+\kappa_{j}-\kappa_{k}>0$ and one of inequalities (7)-(9) is violated. Thus, $\Phi_{t}=p_{1} \Phi_{t}^{(1)}+$ $p_{2} \Phi_{t}^{(2)}+p_{3} \Phi_{t}^{(3)}$ is eternal CP indivisible.

Thus, we have constructed a two-parameter family (since $\left.p_{1}+p_{2}+p_{3}=1\right)$ of eternal CP indivisible processes as a mixture of three ultimate $\mathrm{CP}$ divisible dynamical maps with clear physical meaning. This family comprises the previously known examples as a partial case when $p_{1}=0$. Corresponding trajectories in the parameter space are depicted in Fig. 7. Note that the constructed family is a mixture of CP divisible processes, so it can be realized by a collision model developed in the previous section.

\section{P DIVISIBILITY}

In this section, we review the elementary operational features of $\mathrm{P}$ divisible dynamical processes.

\section{A. Probability of confusion}

Consider a positive map $\Theta ;{ }^{1}$ then the quantum relative entropy $S(\varrho \| \sigma)=\operatorname{tr}[\varrho(\ln \varrho-\ln \sigma)]$ is a monotone under

\footnotetext{
${ }^{1} \mathrm{~A}$ positive map is a linear map that transforms positive semidefinite operators into positive semidefinite ones. Hereafter, we assume the
} 


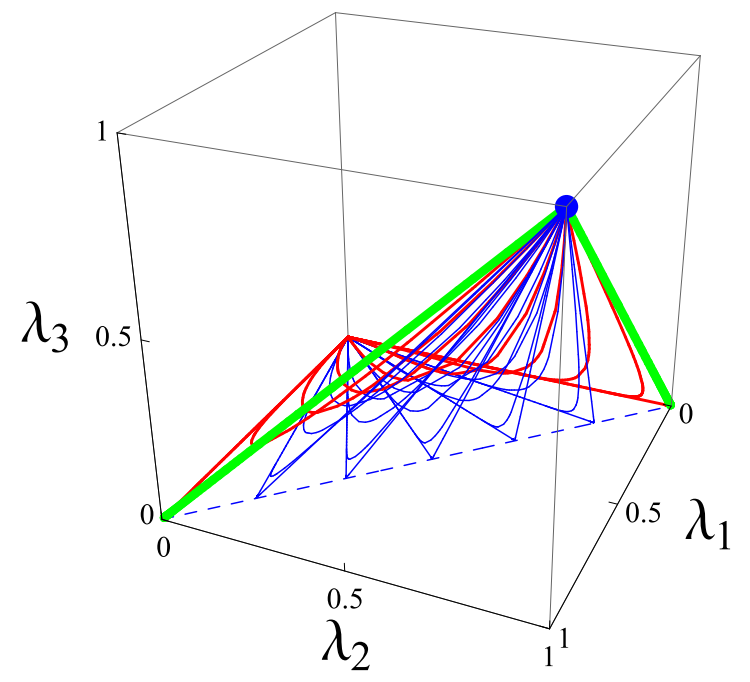

FIG. 7. Trajectories of eternal CP indivisible Pauli dynamical maps (thin blue lines), which are convex mixtures of pure dephasing processes (thick straight green lines) and one of nontrivial ultimate $\mathrm{CP}$ divisible maps (one of thick curved red lines).

positive maps [74], i.e.,

$$
S(\Theta[\varrho] \| \Theta[\sigma]) \leqslant S(\varrho \| \sigma),
$$

for all density matrices $\varrho$ and $\sigma$. On the other hand, the quantum analog of Sanov's theorem [75] states that the probability of confusing two quantum states $\varrho$ and $\sigma$ after performing $n$ measurements on $\sigma$ equals

$$
P_{n}(\sigma \rightarrow \varrho)=e^{-n S(\varrho \| \sigma)} \quad \text { if } n \gg 1 .
$$

Therefore, the probability of confusing two states $\varrho$ and $\sigma$ monotonically increases in $\mathrm{P}$ divisible processes $[S(\varrho \| \sigma)$ monotonically decreases].

\section{B. Distinguishability}

The trace distance $D(\varrho, \sigma)=\frac{1}{2}\|\varrho-\sigma\|_{1}$ between qubit states $\varrho$ and $\sigma$ is a monotone under qubit positive maps $\Theta$ too, i.e.,

$$
D(\Theta[\varrho], \Theta[\sigma]) \leqslant D(\varrho, \sigma) .
$$

On the other hand, the trace distance quantifies the probability of successful discrimination of quantum states $\varrho$ and $\sigma$ in a single-shot measurement. For P divisible processes, this probability monotonically decreases.

\section{Classical capacity}

If the process $\Phi_{t}$ is unital, then the map $\Theta_{t, t+s}$ is also unital. The classical capacity $C$ of a qubit unital channel reads $C\left(\Phi_{t}\right)=1-h_{2}\left[\frac{1}{2}\left(1-\max \left(\left|\lambda_{1}(t)\right|,\left|\lambda_{2}(t)\right|,\left|\lambda_{3}(t)\right|\right)\right)\right]$, where $h_{2}(x)=-x \log _{2} x-(1-x) \log _{2}(1-x)$. It is not hard to see that all $\left|\lambda_{i}(t)\right|, i=1,2,3$, monotonically decrease if $\Theta_{t, t+s}$ is

dimensions of the input and the output spaces to be equal. Also, for the sake of brevity, we will refer to positive trace-preserving maps as positive. positive for all $t, s$. Therefore, if the qubit unital process $\Phi_{t}$ is P divisible, then its classical capacity $C\left(\Phi_{t}\right)$ monotonically decreases with time $t$.

\section{Separability}

If a positive map $\Theta$ is applied to a part of separable state $R=$ $\sum_{i} \pi_{i} \varrho_{i} \otimes \sigma_{i}, \pi_{i} \geqslant 0$, then its separability is preserved since $(\Theta \otimes \mathrm{Id})[R]=\sum_{i} \pi_{i} \Theta\left[\varrho_{i}\right] \otimes \sigma_{i}$ is a valid density operator. Thus, if the process $\Phi_{t}$ is $\mathrm{P}$ divisible, then its action on a part of a composite system cannot result in the revival of entanglement.

Suppose that by time $t=t_{\mathrm{EB}}$, the process $\Phi_{t}$ becomes entanglement breaking [76,77], i.e., $\Phi_{t_{\mathrm{EB}}}$ is effectively a measure-and-prepare procedure (quantum-classical-quantum channel) of the Holevo form $\Phi_{t \mathrm{~EB}}[\varrho]=\sum_{k} \operatorname{tr}\left[\varrho E_{k}\right] \varrho_{k}$, where $\left\{E_{k}\right\}$ is a positive operator-valued measure. If $\Phi_{t}$ acts on a part of a composite system (initially in the state $R_{0}$ ), then $\left(\Phi_{t_{\mathrm{EB}}} \otimes \mathrm{Id}\right)\left[R_{0}\right]$ is separable and the further P divisible dynamics leaves this state separable.

Suppose the channel $\Phi_{t} \otimes \Phi_{t}$ becomes entanglement annihilating [78,79] by time $t=t_{\mathrm{EA}}$, and $\Phi_{t}$ is P divisible for $t>$ $t_{\mathrm{EA}}$. Then, $\left(\Phi_{t_{\mathrm{EA}}} \otimes \Phi_{t_{\mathrm{EA}}}\right)\left[R_{0}\right]$ is separable and $\left(\Phi_{t} \otimes \Phi_{t}\right)\left[R_{0}\right]$ remains separable for $t>t_{\mathrm{EA}}$.

For instance, the Pauli channel $\Phi$ with parameters $\lambda_{1}, \lambda_{2}, \lambda_{3}$ results in entanglement-annihilating channel $\Phi \otimes \Phi$ if and only if $\lambda_{1}^{2}+\lambda_{2}^{2}+\lambda_{3}^{2} \leqslant 1$ [79]. The process $\Phi_{t}=p_{1} e^{\mathcal{L}_{1} t}+$ $p_{2} e^{\mathcal{L}_{2} t}+p_{3} e^{\mathcal{L}_{3} t}$ with dissipators $\mathcal{L}_{i}[\varrho]=\gamma\left(\sigma_{i} \varrho \sigma_{i}-\varrho\right)$ becomes entanglement annihilating if

$$
p_{1}^{2}+p_{2}^{2}+p_{3}^{2}=\frac{1-e^{-\gamma t_{\mathrm{EA}}}-e^{-2 \gamma t_{\mathrm{EA}}}}{1-e^{-\gamma t_{\mathrm{EA}}}+e^{-2 \gamma t_{\mathrm{EA}}}} .
$$

Positive divisibility of the map $\Phi_{t}$ guarantees separability of $\left(\Phi_{t} \otimes \Phi_{t}\right)\left[R_{0}\right]$ for all $t>t_{\mathrm{EA}}$.

\section{E. Tensor power}

Clearly, a map $\Theta \otimes \Theta$ can be nonpositive even if $\Theta$ is positive [80,81]. Thus, even if $\Phi_{t}$ is $\mathrm{P}$ divisible, $\Phi_{t} \otimes \Phi_{t}$ can still be P indivisible. However, if $\Phi_{t} \otimes \Phi_{t}$ is P divisible, then $\Phi_{t}$ is CP divisible [65].

\section{COLLISION MODELS FOR P INDIVISIBLE DYNAMICAL MAPS}

P indivisible (essentially non-Markovian) dynamical maps $\Phi_{t}$ can exhibit properties opposite to those described in the previous section, namely, the probability of confusion of two states, distinguishability of states, and classical capacity can be nonmonotonic functions of time. In the following sections, we construct collision models of specific and general $P$ indivisible processes and present an example of the dynamical map, which monotonically shrinks the volume of accessible states but is not $\mathrm{P}$ divisible.

\section{A. Essentially non-Markovian dephasing process}

As an example of $\mathrm{P}$ indivisible dynamics, consider a correlated environment of $n$ qubits (Fig. 8) in the state

$$
\Xi=\frac{1}{2}\left(\left|0^{\otimes n}\right\rangle\left\langle 0^{\otimes n}|+| 1^{\otimes n}\right\rangle\left\langle 1^{\otimes n}\right|\right) .
$$




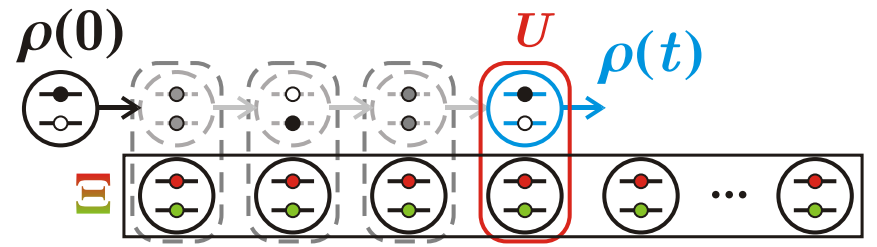

FIG. 8. Essentially non-Markovian (P indivisible) dynamics in a collision model with correlated environment. Correlations are encoded in color (upper and lower levels): either all environment qubits are excited (red, upper level) or they are all in a ground state (green, lower level).

The elementary unitary transformation $U_{k}$ describes the evolution of the system and $k$ th environment qubit. Suppose $U_{k}=e^{i g \tau \sigma_{z}} \otimes|0\rangle_{k}\left\langle 0\left|+e^{-i g \tau \sigma_{z}} \otimes\right| 1\right\rangle_{k}\langle 1| ;$ then, after $n=\frac{t}{\tau}$ collisions, we get

$$
\begin{aligned}
\Phi_{t}^{z}[\varrho] & =\operatorname{tr}_{\mathrm{env}}\left[U_{n} \cdots U_{2} U_{1} \varrho \otimes \Xi U_{1}^{\dagger} U_{2}^{\dagger} \cdots U_{n}^{\dagger}\right] \\
& =\cos ^{2}(n g \tau) \varrho+\sin ^{2}(n g \tau) \sigma_{z} \varrho \sigma_{z} \\
& =\cos ^{2}(g t) \varrho+\sin ^{2}(g t) \sigma_{z} \varrho \sigma_{z} .
\end{aligned}
$$

Clearly, the resulting dephasing dynamics $\Phi_{t}$ is P divisible if $0<g t<\frac{\pi}{4}$ and $\mathrm{P}$ indivisible if $\frac{\pi}{4}<g t<\frac{\pi}{2}$. Then the periods of $\mathrm{P}$ divisibility and $\mathrm{P}$ indivisibility alternate. The information about the initial system state $\varrho$ is stored in the environment when the process is $\mathrm{P}$ divisible, and the backflow of information occurs when the process is $\mathrm{P}$ indivisible.

\section{B. Mixture of essentially non-Markovian dephasing processes}

Similarly to the previous section, $\mathrm{P}$ indivisible dephasing processes $\Phi_{t}^{x}$ and $\Phi_{t}^{y}$ along the $x$ and $y$ axes of the Bloch ball can be achieved by collision models. The mixture $\Phi_{t}=p_{1} \Phi_{t}^{x}+p_{2} \Phi_{t}^{y}+p_{3} \Phi_{t}^{z}$ can be realized with a correlated environment made of $n$ six-level systems in the state

$$
\Xi=\sum_{m=1,2,3} \frac{p_{m}}{2}\left(\left|0_{m}^{\otimes n}\right\rangle\left\langle 0_{m}^{\otimes n}|+| 1_{m}^{\otimes n}\right\rangle\left\langle 1_{m}^{\otimes n}\right|\right),
$$

where $m$ labels the pairs of levels (effective qubit states $\left|0_{m}\right\rangle$ and $\left.\left|1_{m}\right\rangle\right)$, and elementary unitary transformations

$$
U_{k}=\sum_{m=1,2,3} e^{i g \tau \sigma_{m}} \otimes\left|0_{m}\right\rangle_{k}\left\langle 0_{m}\left|+e^{-i g \tau \sigma_{m}} \otimes\right| 1_{m}\right\rangle_{k}\left\langle 1_{m}\right| .
$$

A pictorial representation of the resulting dynamical map,

$$
\begin{aligned}
\Phi_{t} & =p_{1} \Phi_{t}^{x}+p_{2} \Phi_{t}^{y}+p_{3} \Phi_{t}^{z} \\
& =\cos ^{2}(\alpha t) \varrho+\sin ^{2}(\alpha t) \sum_{m=1}^{3} p_{m} \sigma_{m} \varrho \sigma_{m},
\end{aligned}
$$

is a straight line in the parameter space $\lambda_{1}, \lambda_{2}, \lambda_{3}$.

For instance, in the case $p_{1}=p_{2}=p_{3}=\frac{1}{3}$, we obtain a depolarizing map $\mathcal{D}_{p(t)}[\varrho]=p(t) \varrho+(1-p(t)) \operatorname{tr}[\varrho] \frac{1}{2} I$ with $p(t)=\frac{1}{3}(1+2 \cos 2 g t)$. In such a process, the Bloch ball gradually shrinks to a point, then extends in inverted form unless its radius equals $\frac{1}{3}$ (the best approximation of universal NOT operation), and then the process goes in the opposite direction until the region of accessible states occupies the whole Bloch ball again; after that, the process is continuous from the very beginning.

\section{Arbitrary pure dephasing process}

In Sec. IX A, we considered a essentially non-Markovian pure dephasing process with the coherence function $\cos (2 g t)$. In this section, we construct a collision model which results in a pure dephasing process with the arbitrary continuous real coherence function $f(t)$ that is bounded $(|f(t)| \leqslant 1)$ and $f(0)=1$.

We start with a dephasing process in the basis of eigenvectors of $\sigma_{z}$, i.e., the density matrix transformation

$$
\left(\begin{array}{ll}
\varrho_{11} & \varrho_{12} \\
\varrho_{21} & \varrho_{22}
\end{array}\right) \rightarrow\left(\begin{array}{cc}
\varrho_{11} & f(t) \varrho_{12} \\
f(t) \varrho_{21} & \varrho_{22}
\end{array}\right),
$$

which corresponds to a trajectory $\lambda(t)=(f(t), f(t), 1)$ in the parameter space.

Consider a correlated environment in the state

$$
\begin{aligned}
\Xi= & \frac{1}{2}\left(\left|i_{1}\right\rangle\left\langle i_{1}|\otimes| i_{2}\right\rangle\left\langle i_{2}|\otimes \cdots \otimes| i_{n}\right\rangle\left\langle i_{n}\right| \otimes \cdots\right. \\
& \left.+\left|\overline{i_{1}}\right\rangle\left\langle\overline{i_{1}}|\otimes| \overline{i_{2}}\right\rangle\left\langle\overline{i_{2}}|\otimes \cdots \otimes| \overline{i_{n}}\right\rangle\left\langle\overline{i_{n}}\right| \otimes \cdots\right) \\
= & \frac{1}{2} \bigotimes_{k}\left|i_{k}\right\rangle\left\langle i_{k}\left|+\frac{1}{2} \bigotimes_{k}\right| \overline{i_{k}}\right\rangle\left\langle\overline{i_{k}}\right|,
\end{aligned}
$$

where either $i_{k}=0$ and $\overline{i_{k}}=1$, or $i_{k}=1$ and $\overline{i_{k}}=0$. Elementary unitary transformations $U_{k}=e^{i g \tau \sigma_{z}} \otimes|0\rangle_{k}\langle 0|+$ $e^{-i g \tau \sigma_{z}} \otimes|1\rangle_{k}\langle 1|$ result in the following dynamical map after $n=\frac{t}{\tau}$ collisions:

$$
\begin{aligned}
\Phi_{t}[\varrho]= & \operatorname{tr}_{\mathrm{env}}\left[U_{n} \cdots U_{2} U_{1} \varrho \otimes \Xi U_{1}^{\dagger} U_{2}^{\dagger} \cdots U_{n}^{\dagger}\right] \\
= & \cos ^{2}\left\{\left[n_{0}(t)-n_{1}(t)\right] g \tau\right\} \varrho \\
& +\sin ^{2}\left\{\left[n_{0}(t)-n_{1}(t)\right] g \tau\right\} \sigma_{z} \varrho \sigma_{z},
\end{aligned}
$$

where $n_{0}(t)=\sum_{k=1}^{n} \delta_{i_{k}, 0}$ and $n_{1}(t)=\sum_{k=1}^{n} \delta_{i_{k}, 1}=n-n_{0}$. Apparently, $\left[n_{0}(t)-n_{1}(t)\right] \tau=2 n_{0}(t) \tau-t$ and

$$
f(t)=\cos \left\{2 g\left[2 n_{0}(t) \tau-t\right]\right\} .
$$

Therefore, to get the desired dynamics, one needs to arrange the number $n_{0}(t)$ of 0 's in indices $i_{k}$ of environment state (55) in accordance with the formula

$$
n_{0}(t) \tau=\frac{\arccos f(t)}{4 g}+\frac{t}{2} .
$$

In the usual continuous limit $\tau \rightarrow 0, g \tau \rightarrow$ const, the left-hand side of Eq. (58) has the meaning of the integral $n_{0}(t) \tau=$ $\int_{0}^{t} w_{0}\left(t^{\prime}\right) d t^{\prime}$, where $w_{0}(t)$ is the probability of encountering 0 at every collision in the first line of the environment state (55). Finally,

$$
w_{0}(t)=-\frac{f^{\prime}(t)}{4 g \sqrt{1-f^{2}(t)}}+\frac{1}{2} .
$$

If $f^{\prime}(t)=0$ when $f(t)=1$, then the right-hand side can be made non-negative and bounded from above by 1 for sufficiently large $g$. If $f^{\prime}(t) \neq 0$ when $f(t)=1$ (as it takes place, e.g., in Markov approximation), one has to resort to the stroboscopic limit and replace $g$ by $\frac{g}{\sqrt{\tau}}$, which enables one to meet the requirement $0 \leqslant w_{0}(t) \leqslant 1$. Similarly, one can construct the processes of arbitrary dephasing in the bases of eigenstates of operators $\sigma_{x}$ and $\sigma_{y}$. 


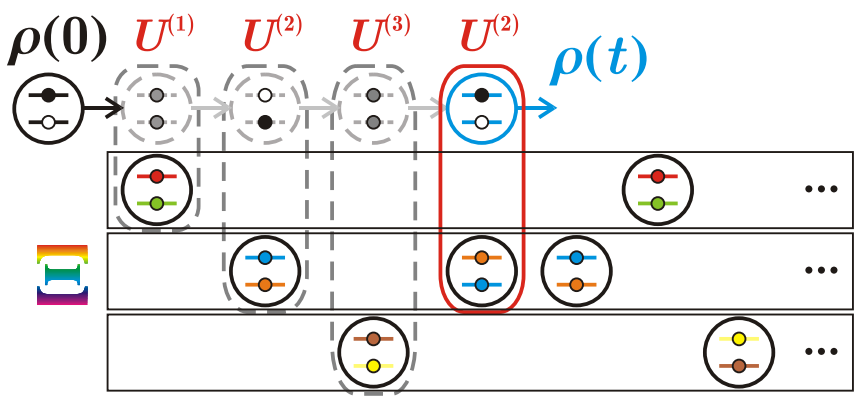

FIG. 9. Collision model simulating essentially non-Markovian Pauli dynamical maps.

\section{Arbitrary Pauli dynamical maps}

In this section, we construct a collision model which is able to reproduce any dynamics $\lambda(t)$ satisfying the condition of complete positivity of the corresponding Pauli dynamical map $\Phi_{t}$. In other words, given a trajectory in the parameter space [Fig. 1(a)], we construct a collision model leading to such a trajectory.

The requirement of complete positivity is automatically fulfilled if the functions $q_{j}(t)$ that are defined through

$$
\left(\begin{array}{l}
q_{0}(t) \\
q_{1}(t) \\
q_{2}(t) \\
q_{3}(t)
\end{array}\right)=\frac{1}{4}\left(\begin{array}{cccc}
1 & 1 & 1 & 1 \\
1 & 1 & -1 & -1 \\
1 & -1 & 1 & -1 \\
1 & -1 & -1 & 1
\end{array}\right)\left(\begin{array}{c}
1 \\
\lambda_{1}(t) \\
\lambda_{2}(t) \\
\lambda_{3}(t)
\end{array}\right)
$$

satisfy $q_{j}(t) \geqslant 0$ for all $j=0,1,2,3$.

To get an arbitrary Pauli dynamical map with non-negative functions $q_{0}(t), q_{1}(t), q_{2}(t), q_{3}(t)$, one needs to combine three (essentially non-Markovian) dephasing processes considered in the previous section. This is achieved with the environment composed of three types of qubits. Denote these types $x, y, z$; then, the $k$ th collision of the system qubit with the $m$ th type of environment qubits is described by the elementary unitary transformation

$$
U_{k}^{(m)}=e^{i g \tau \sigma_{m}} \otimes|0\rangle_{k}\left\langle 0\left|+e^{-i g \tau \sigma_{m}} \otimes\right| 1\right\rangle_{k}\langle 1| .
$$

Qubits of the same kind are correlated, so the total environment state reads

$$
\Xi=\bigotimes_{m=x, y, z}\left(\frac{1}{2} \bigotimes_{k \in\left\{k_{m}\right\}}\left|i_{k}\right\rangle\left\langle i_{k}\left|+\frac{1}{2} \bigotimes_{k \in\left\{k_{m}\right\}}\right| \overline{i_{k}}\right\rangle\left\langle\overline{i_{k}}\right|\right),
$$

where $\left\{k_{x}\right\},\left\{k_{y}\right\},\left\{k_{z}\right\}$ are subsequences of collision numbers $k \in \mathbb{N}$ such that $\left\{k_{m}\right\} \cap\left\{k_{m^{\prime}}\right\}=\emptyset$ if $m \neq m^{\prime}$ and $\left\{k_{x}\right\} \cup\left\{k_{y}\right\} \cup$ $\left\{k_{z}\right\}=\mathbb{N}$. The physics of such collisions is depicted in Fig. 9.

The resulting map is

$$
\Phi_{t}[\varrho]=\operatorname{tr}_{\mathrm{env}}\left[U_{n} \cdots U_{1}(\varrho \otimes \Xi) U_{1}^{\dagger} \cdots U_{n}^{\dagger}\right]
$$

where $U_{k}=U_{k}^{\left(m: k_{m}=k\right)}$. The intermediate map $\Theta_{t, t+\tau}=$ $\Phi_{t+\tau} \circ \Phi_{t}^{-1}$ between collisions realizes one of the infinitesimal maps $\Theta_{t, t+\tau}^{(x)}, \Theta_{t, t+\tau}^{(y)}$, and $\Theta_{t, t+\tau}^{(z)}$. Collision with the $k_{m}$ th particle results in the map $\Theta_{t, t+\tau}^{(m)}$. Clearly, for a fixed $m$, the product $\prod_{k_{m}} \Theta_{k_{m} \tau,\left(k_{m}+1\right) \tau}^{(m)}=\Phi_{t}^{(m)}$ is simply the dephasing map in the eigenbasis of operator $\sigma_{m}$ with dephasing function $f_{m}(t)$ given by a modification of Eq. (57),

$$
f_{m}(t)=\cos \left\{2\left[n_{0}^{(m)}-n_{1}^{(m)}\right] g \tau\right\},
$$

where $n_{0}^{(m)}=\sum_{k_{m} \leqslant n} \delta_{i_{k_{m}}, 0}$ and $n_{1}^{(m)}=\sum_{k_{m} \leqslant n} \delta_{i_{k_{m}}, 1}$. All physical functions $f_{m}(t), m=1,2,3$, can be realized in the usual continuous or stroboscopic limit as it was demonstrated for a single dephasing map. Then a sequence of collisions with different types of qubits during a short time $d t(d t \gg \tau)$ results in the product

$$
\Theta_{t, t+d t}=\Theta_{t, t+d t}^{(x)} \Theta_{t, t+d t}^{(y)} \Theta_{t, t+d t}^{(z)} .
$$

Note that all $\Theta_{t, t+d t}^{(m)}$ commute. Consequently, the parameters $\lambda_{1}(t), \lambda_{2}(t)$, and $\lambda_{3}(t)$ of the map $\Phi_{t}$ satisfy differential equations $\lambda_{1}^{\prime}(t)=f_{2}^{\prime}(t)+f_{3}^{\prime}(t), \lambda_{2}^{\prime}(t)=f_{1}^{\prime}(t)+$ $f_{3}^{\prime}(t)$, and $\lambda_{3}^{\prime}(t)=f_{1}^{\prime}(t)+f_{2}^{\prime}(t)$, from which it follows that $\lambda_{1}(t)=f_{2}(t)+f_{3}(t)-1, \lambda_{2}(t)=f_{1}(t)+f_{3}(t)-1$, and $\lambda_{3}(t)=f_{1}(t)+f_{2}(t)-1$. Finally, using Eq. (60), we find the explicit form of the functions $f_{m}(t)=1-2 q_{m}(t)$.

The algorithm for producing arbitrary dynamics $\lambda(t)$ in parameter space is the following. Calculate $q_{m}(t)$ by Eq. (60) and $f_{m}(t)=1-2 q_{m}(t)$. For each $m$, distribute 0 's and 1's in accordance with formula (64). Create the correlated state (62) with the corresponding distributions of 0's and 1's in the $m$ th branch. Let the system qubit interact with environment qubits of type $m$ according to the elementary evolution operator (61).

\section{E. Dynamical maps shrinking the volume of accessible states}

One more approach to the characterization of nonMarkovianity is based on the quantification of the volume of accessible states [18]. Using the metric induced by the HilbertSchmidt distance for qubit states, the volume of accessible states of a qubit dynamical map $\Phi_{t}$ is simply the volume of the ellipsoid in the Bloch ball picture, which corresponds to the domain of $\Phi_{t}$. For Pauli dynamical maps $\Phi_{t}$ of the form (2), the volume of accessible states $V(t)=\left|\lambda_{1}(t) \lambda_{2}(t) \lambda_{3}(t)\right|$. A process $\Phi_{t}$ monotonically shrinks the volume of accessible states if any intermediate map $\Theta_{t, t+s}$ does so.

Let us present an example of the Pauli dynamical map $\Phi_{t}$ which monotonically shrinks the volume of accessible states but is not $\mathrm{P}$ divisible. Let $\lambda_{1}(t)=e^{-2 t}\left(1-\frac{1}{10}[1-\right.$ $\cos 40 t]), \lambda_{2}(t)=e^{-2 t}\left(1-\frac{1}{10} \sin 40 t\right), \lambda_{3}(t)=e^{-4 t}$; then, $\Phi_{t}$ is a physical process indeed since all $q_{i}(t) \geqslant 0$ and can be realized via a collision model with correlated environment (see the preceding section). It is not hard to see that the volume of accessible states $V(t)$ monotonically decreases, whereas both $\lambda_{1}(t)$ and $\lambda_{2}(t)$ are not monotonic, i.e., the process is not $\mathrm{P}$ divisible (see the trajectory and the corresponding $\kappa$ vector in Fig. 10).

\section{CONCLUSIONS}

We have studied the relation between different forms of divisibility of dynamical maps and collision models that stroboscopically simulate such dynamical maps. Our findings are illustrated by Pauli dynamical maps, which allow a particularly visual pictorial representation of process trajectories in the parameter space. 


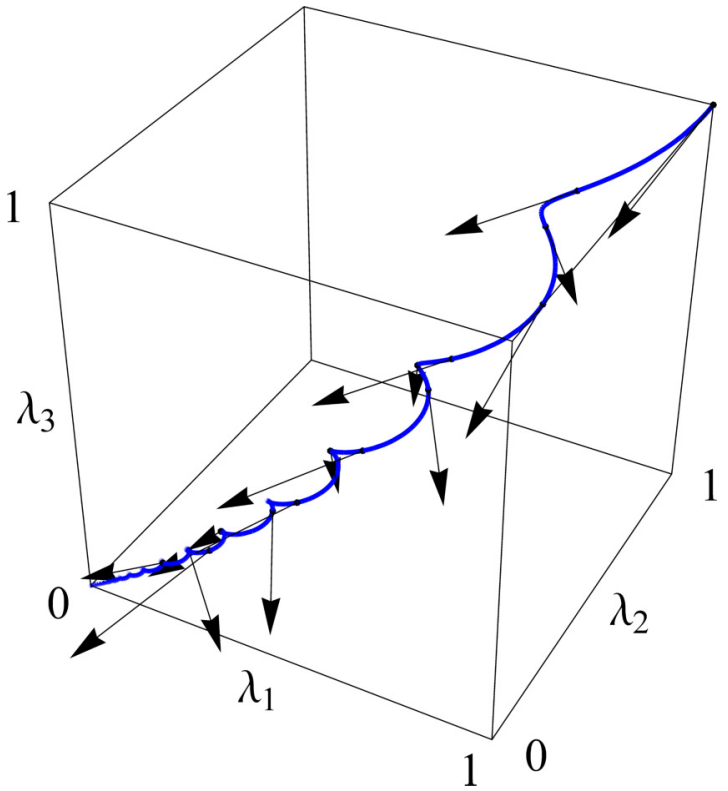

FIG. 10. Trajectory of the Pauli dynamical map, which is not $\mathrm{P}$ divisible but monotonically shrinks the volume of accessible states. Arrows represent directions of the $\kappa$ vector at particular time moments.

A concept of ultimate $\mathrm{CP}$ divisible maps has been introduced: ultimate CP divisible processes can be understood as ultimate dynamical maps still simulable by collision models with factorized environment. Ultimate CP divisible semigroups of Pauli maps are fully characterized, with the interaction Hamiltonian being specified.

Within the framework of collision models, we have demonstrated additivity and multiplicativity of time-dependent generators of $\mathrm{CP}$ divisible processes. The environment remains factorized in this case. Roughly speaking, to realize a weighted sum of generators of $\mathrm{CP}$ divisible maps, one has to shuffle individual environments using a tensor product.

Using correlated environment states, we have explicitly constructed a collision model realizing the mixture of $\mathrm{CP}$ divisible maps. The latter technique was used to simulate a two-parameter family of eternal $\mathrm{CP}$ indivisible maps. This family represents a mixture of two pure dephasing processes and a skewed version of the generalized amplitude damping process. Continuing the rough analogy, a mixture of dynamical maps corresponds to uniting individual environments via direct sum operation.

Also, we have reviewed general properties of $\mathrm{P}$ divisible dynamical maps. In particular, using a quantum analog of Sanov's theorem, we have noticed that the probability of confusing two states monotonically increases in $\mathrm{P}$ divisible processes. As far as $\mathrm{P}$ indivisible processes are concerned, we have explicitly constructed collision models simulating arbitrary Pauli dynamical maps.

\section{ACKNOWLEDGMENTS}

This research was initiated during the first Quantum Physics Unconference organized in Lapland in 2015 by S.M., M.Z., and Teiko Heinosaari. The study is supported by the Russian Science Foundation under Project No. 16-11-00084 and performed at the Moscow Institute of Physics and Technology. S.M. and J.P. acknowledge financial support from the Horizon 2020 EU collaborative projects QuProCS (Grant Agreement No. 641277), the Academy of Finland (Project No. 287750), and the Magnus Ehrnrooth Foundation. M.Z. acknowledges the support of Slovak research and development agency Project No. APVV-14-0878 (QETWORK), Slovak Academy of Sciences grant agency VEGA Project No. 2/0173/17 (MAXAP), and Grant agency of Czech Republic Project No. GA16-22211S.
[1] A. S. Holevo and V. Giovannetti, Quantum channels and their entropic characteristics, Rep. Prog. Phys. 75, 046001 (2012).

[2] G. M. Palma, K.-A. Suominen, and A. K. Ekert, Quantum computers and dissipation, Proc. R. Soc. London A 452, 567 (1996).

[3] L. Aolita, F. de Melo, and L. Davidovich, Open-system dynamics of entanglement: A key issues review, Rep. Prog. Phys. 78, 042001 (2015).

[4] Á. Rivas, S. F. Huelga, and M. B. Plenio, Quantum nonMarkovianity: Characterization, quantification and detection, Rep. Prog. Phys. 77, 094001 (2014).

[5] H.-P. Breuer, E.-M. Laine, J. Piilo, and B. Vacchini, Colloquium: Non-Markovian dynamics in open quantum systems, Rev. Mod. Phys. 88, 021002 (2016).

[6] I. de Vega and D. Alonso, Dynamics of non-Markovian open quantum systems, Rev. Mod. Phys. 89, 015001 (2017).

[7] B.-H. Liu, L. Li, Y.-F. Huang, C.-F. Li, G.-C. Guo, E.-M. Laine, H.-P. Breuer, and J. Piilo, Experimental control of the transition from Markovian to non-Markovian dynamics of open quantum systems, Nat. Phys. 7, 931 (2011).

[8] J.-S. Tang, C.-F. Li, Y.-L. Li, X.-B. Zou, G.-C. Guo, H.-P. Breuer, E.-M. Laine, and J. Piilo, Measuring non-Markovianity of processes with controllable system-environment interaction, Europhys. Lett. 97, 10002 (2012).

[9] A. Chiuri, C. Greganti, L. Mazzola, M. Paternostro, and P. Mataloni, Linear optics simulation of quantum non-Markovian dynamics, Sci. Rep. 2, 968 (2012).

[10] S. Gröblacher, A. Trubarov, N. Prigge, G. D. Cole, M. Aspelmeyer, and J. Eisert, Observation of non-Markovian micromechanical Brownian motion, Nat. Commun. 6, 7606 (2015).

[11] N. K. Bernardes, A. Cuevas, A. Orieux, C. H. Monken, P. Mataloni, F. Sciarrino, and M. F. Santos, Experimental observation of weak non-Markovianity, Sci. Rep. 5, 17520 (2015)

[12] N. K. Bernardes, J. P. S. Peterson, R. S. Sarthour, A. M. Souza, C. H. Monken, I. Roditi, I. S. Oliveira, and M. F. Santos, 
High resolution non-Markovianity in NMR, Sci. Rep. 6, 33945 (2016).

[13] S. Cialdi, M. A. C. Rossi, C. Benedetti, B. Vacchini, D. Tamascelli, S. Olivares, and M. G. A. Paris, All-optical quantum simulator of qubit noisy channels, Appl. Phys. Lett. 110, 081107 (2017).

[14] H.-P. Breuer, E.-M. Laine, and J. Piilo, Measure for the Degree of Non-Markovian Behavior of Quantum Processes in Open Systems, Phys. Rev. Lett. 103, 210401 (2009).

[15] E.-M. Laine, J. Piilo, and H.-P. Breuer, Measure for the nonMarkovianity of quantum processes, Phys. Rev. A 81, 062115 (2010).

[16] M. M. Wolf, J. Eisert, T. S. Cubitt, and J. I. Cirac, Assessing NonMarkovian Quantum Dynamics, Phys. Rev. Lett. 101, 150402 (2008).

[17] Á. Rivas, S. F. Huelga, and M. B. Plenio, Entanglement and Non-Markovianity of Quantum Evolutions, Phys. Rev. Lett. 105, 050403 (2010).

[18] S. Lorenzo, F. Plastina, and M. Paternostro, Geometrical characterization of non-Markovianity, Phys. Rev. A 88, 020102(R) (2013).

[19] X.-M. Lu, X. Wang, and C. P. Sun, Quantum Fisher information flow and non-Markovian processes of open systems, Phys. Rev. A 82, 042103 (2010).

[20] S. Luo, S. Fu, and H. Song, Quantifying non-Markovianity via correlations, Phys. Rev. A 86, 044101 (2012).

[21] B. Bylicka, D. Chruściński, and S. Maniscalco, NonMarkovianity and reservoir memory of quantum channels: A quantum information theory perspective, Sci. Rep. 4, 5720 (2014).

[22] H. S. Dhar, M. N. Bera, and G. Adesso, Characterizing nonMarkovianity via quantum interferometric power, Phys. Rev. A 91, 032115 (2015).

[23] M. M. Wolf and J. I. Cirac, Dividing quantum channels, Commun. Math. Phys. 279, 147 (2008).

[24] N. Megier, D. Chruściński, J. Piilo, and W. T. Strunz, Eternal non-Markovianity: From random unitary to Markov chain realisations, Sci. Rep. 7, 6379 (2017).

[25] M. J. W. Hall, J. D. Cresser, L. Li, and E. Andersson, Canonical form of master equations and characterization of non-Markovianity, Phys. Rev. A 89, 042120 (2014).

[26] B. Bylicka, M. Johansson, and A. Acín, Constructive Method for Detecting the Information Backflow of Non-Markovian Dynamics, Phys. Rev. Lett. 118, 120501 (2017).

[27] D. Chruściński and S. Maniscalco, Degree of Non-Markovianity of Quantum Evolution, Phys. Rev. Lett. 112, 120404 (2014).

[28] F. A. Wudarski, P. Należyty, G. Sarbicki, and D. Chruściński, Admissible memory kernels for random unitary qubit evolution, Phys. Rev. A 91, 042105 (2015).

[29] D. Chruściński and F. A. Wudarski, Non-Markovianity degree for random unitary evolution, Phys. Rev. A 91, 012104 (2015).

[30] D. Chruściński and K. Siudzińska, Generalized Pauli channels and a class of non-Markovian quantum evolution, Phys. Rev. A 94, 022118 (2016).

[31] M. J. W. Hall, Complete positivity for time-dependent qubit master equations, J. Phys. A: Math. Theor. 41, 205302 (2008).

[32] H.-P. Breuer and F. Petruccione, The Theory of Open Quantum Systems (Oxford University Press, Oxford, 2002).

[33] J. Rau, Relaxation phenomena in spin and harmonic oscillator systems, Phys. Rev. 129, 1880 (1963).
[34] V. Scarani, M. Ziman, P. Štelmachovič, N. Gisin, and V. Bužek, Thermalizing Quantum Machines: Dissipation and Entanglement, Phys. Rev. Lett. 88, 097905 (2002).

[35] M. Ziman, P. Štelmachovič, V. Bužek, M. Hillery, V. Scarani, and N. Gisin, Diluting quantum information: An analysis of information transfer in system-reservoir interactions, Phys. Rev. A 65, 042105 (2002).

[36] M.Ziman and V. Bužek, in Quantum Dynamics and Information, edited by R. Olkiewicz et al. (World Scientific, Singapore, 2011), pp. 199-227.

[37] D. Kretschmann and R. F. Werner, Quantum channels with memory, Phys. Rev. A 72, 062323 (2005).

[38] V. Giovannetti, A dynamical model for quantum memory channels, J. Phys. A: Math. Gen. 38, 10989 (2005).

[39] V. Giovannetti and G. M. Palma, Master Equations for Correlated Quantum Channels, Phys. Rev. Lett. 108, 040401 (2012).

[40] F. Caruso, V. Giovannetti, C. Lupo, and S. Mancini, Quantum channels and memory effects, Rev. Mod. Phys. 86, 1203 (2014).

[41] T. Rybár, S. N. Filippov, M. Ziman, and V. Bužek, Simulation of indivisible qubit channels in collision models, J. Phys. B 45, 154006 (2012).

[42] N. K. Bernardes, A. R. R. Carvalho, C. H. Monken, and M. F. Santos, Environmental correlations and Markovian to non-Markovian transitions in collisional models, Phys. Rev. A 90, 032111 (2014).

[43] N. K. Bernardes, A. R. R. Carvalho, C. H. Monken, and M. F. Santos, Coarse graining a non-Markovian collisional model, Phys. Rev. A 95, 032117 (2017).

[44] A. Dąbrowska, G. Sarbicki, and D. Chruściński, Quantum trajectories for a system interacting with environment in a single photon state: Counting and diffusive processes, arXiv:1706.07967.

[45] C. Pellegrini and F. Petruccione, Non-Markovian quantum repeated interactions and measurements, J. Phys. A: Math. Theor. 42, 425304 (2009).

[46] A. Bodor, L. Diósi, Z. Kallus, and T. Konrad, Structural features of non-Markovian open quantum systems using quantum chains, Phys. Rev. A 87, 052113 (2013).

[47] F. Ciccarello, G. M. Palma, and V. Giovannetti, Collision-modelbased approach to non-Markovian quantum dynamics, Phys. Rev. A 87, 040103(R) (2013).

[48] F. Ciccarello and V. Giovannetti, A quantum non-Markovian collision model: Incoherent swap case, Phys. Scr. T153, 014010 (2013).

[49] R. McCloskey and M. Paternostro, Non-Markovianity and system-environment correlations in a microscopic collision model, Phys. Rev. A 89, 052120 (2014).

[50] S. Kretschmer, K. Luoma, and W. T. Strunz, Collision model for non-Markovian quantum dynamics, Phys. Rev. A 94, 012106 (2016).

[51] A. A. Budini, Embedding non-Markovian quantum collisional models into bipartite Markovian dynamics, Phys. Rev. A 88, 032115 (2013).

[52] S. Lorenzo, F. Ciccarello, and G. M. Palma, Class of exact memory-kernel master equations, Phys. Rev. A 93, 052111 (2016).

[53] L. Hartmann, J. Calsamiglia, W. Dür, and H. J. Briegel, Spin gases as microscopic models for non-Markovian decoherence, Phys. Rev. A 72, 052107 (2005). 
[54] M. Koniorczyk, Á. Varga, P. Rapčan, and V. Bužek, Quantum homogenization and state randomization in semiquantal spin systems, Phys. Rev. A 77, 052106 (2008).

[55] B. Vacchini, Generalized Master Equations Leading to Completely Positive Dynamics, Phys. Rev. Lett. 117, 230401 (2016).

[56] L. Diósi, Non-Markovian open quantum systems: Input-output fields, memory, and monitoring, Phys. Rev. A 85, 034101 (2012).

[57] S. Lorenzo, F. Ciccarello, and G. M. Palma, Composite quantum collision models, Phys. Rev. A 96, 032107 (2017).

[58] S. Cusumano, A. Mari, and V. Giovannetti, Interferometric quantum cascade systems, Phys. Rev. A 95, 053838 (2017).

[59] S. Lorenzo, F. Ciccarello, G. M. Palma, and B. Vacchini, Quantum non-Markovian piecewise dynamics from collision models, arXiv:1706.09025.

[60] C. Lupo, V. Giovannetti, and S. Mancini, Memory effects in attenuation and amplification quantum processes, Phys. Rev. A 82, 032312 (2010).

[61] J. Jin, V. Giovannetti, R. Fazio, F. Sciarrino, P. Mataloni, A. Crespi, and R. Osellame, All-optical non-Markovian stroboscopic quantum simulator, Phys. Rev. A 91, 012122 (2015).

[62] S. Lorenzo, R. McCloskey, F. Ciccarello, M. Paternostro, and G. M. Palma, Landauer's Principle in Multipartite Open Quantum System Dynamics, Phys. Rev. Lett. 115, 120403 (2015).

[63] S. Attal and Y. Pautrat, From repeated to continuous quantum interactions, Ann. Henri Poincaré 7, 59 (2006).

[64] J. Kołodyński, J. Bohr Brask, M. Perarnau-Llobet, and B. Bylicka, Adding dynamical generators in quantum master equations, arXiv:1704.08702.

[65] F. Benatti, D. Chruściński, and S. Filippov, Tensor power of dynamical maps and positive versus completely positive divisibility, Phys. Rev. A 95, 012112 (2017).

[66] M. B. Ruskai, S. Szarek, and E. Werner, An analysis of completely-positive trace-preserving maps on $M_{2}$, Linear Algebra Appl. 347, 159 (2002).

[67] I. Bengtsson and K. Życzkowski, Geometry of Quantum States. An Introduction to Quantum Entanglement (Cambridge University Press, New York, 2006).
[68] V. Gorini, A. Kossakowski, and E. C. G. Sudarshan, Completely positive dynamical semigroups of $N$-level systems, J. Math. Phys. 17, 821 (1976).

[69] G. Lindblad, On the generators of quantum dynamical semigroups, Commun. Math. Phys. 48, 119 (1976).

[70] M. A. Nielsen and I. L. Chuang, Quantum Computation and Quantum Information (Cambridge University Press, Cambridge, 2000).

[71] I. A. Luchnikov and S. N. Filippov, Quantum evolution in the stroboscopic limit of repeated measurements, Phys. Rev. A 95, 022113 (2017).

[72] D. Kretschmann, D. Schlingemann, and R. F. Werner, A continuity theorem for Stinespring's dilation, J. Funct. Anal. 255, 1889 (2008).

[73] L. Ferialdi, Exact non-Markovian master equation for the spin-boson and Jaynes-Cummings models, Phys. Rev. A 95, 020101(R) (2017); 95, 069908(E) (2017).

[74] A. Müller-Hermes and D. Reeb, Monotonicity of the quantum relative entropy under positive maps, Ann. Henri Poincare 18, 1777 (2017).

[75] V. Vedral, M. B. Plenio, K. Jacobs, and P. L. Knight, Statistical inference, distinguishability of quantum states, and quantum entanglement, Phys. Rev. A 56, 4452 (1997).

[76] A. S. Holevo, Quantum coding theorems, Russ. Math. Surveys 53, 1295 (1998).

[77] M. Horodecki, P. W. Shor, and M. B. Ruskai, Entanglement breaking channels, Rev. Math. Phys. 15, 629 (2003).

[78] L. Moravčíková and M. Ziman, Entanglement-annihilating and entanglement-breaking channels, J. Phys. A: Math. Theor. 43, 275306 (2010).

[79] S. N. Filippov, T. Rybár, and M. Ziman, Local two-qubit entanglement-annihilating channels, Phys. Rev. A 85, 012303 (2012).

[80] A. Müller-Hermes, D. Reeb, and M. M. Wolf, Positivity of linear maps under tensor powers, J. Math. Phys. 57, 015202 (2016).

[81] S. N. Filippov and K. Yu. Magadov, Positive tensor products of maps and $n$-tensor-stable positive qubit maps, J. Phys. A: Math. Theor. 50, 055301 (2017). 